\title{
Velocity Inversion by Coherency Optimization ${ }^{1}$
}

by

William W. Symes

Technical Report 88-4, April 1988

${ }^{1}$ Research supported in part by the Office of Naval Research under contract N00014-85-K0725 and by the National Science Foundation under grant DMS86-03164. 



\title{
Velocity Inversion by Coherency Optimization *
}

\author{
William W. Symes
}

May 1988

\begin{abstract}
We introduce an approach to velocity and reflectivity estimation based on optimizing the coherence of multiple shot-gather inversions of reflection seismograms. The resulting algorithm appears to avoid the severe convergence difficulties reported for output (nonlinear) least-squares inversion. We describe in detail an algorithm appropriate for the layered acoustic model, using the convolutional model of the plane-wave ( $p$-tau) seismogram. We give a theoretical analysis and numerical evidence that coherency optimization, as defined here, yields stable and reasonably accurate estimates of both velocity trend and reflectivity, by exploiting reflection phase moveout and amplitudes in a computationally efficient way. We also indicate how the approach may be modified to apply to laterally heterogeneous acoustic models, and (more briefly) to determination of elastic models and source parameters as well.
\end{abstract}

\section{Introduction}

Multi-offset model-based inversion is of interest in the processing of wide-angle seismic reflection data, both for structural information and for the extraction of reflection characteristics directly indicating the presence of hydrocarbon deposits. Almost by definition, techniques designed to extract such informationray-trace velocity estimation, amplitude-preserving before-stack migrationhave the character of partial inversion algorithms, and analysis often reveals that the relation is quite deep.

Inversion algorithms based on the full seismic waveform have been discussed extensively over the past several years; see e.g. Tarantola (1986), Gauthier et al. (1986), MacAulay (1985), Mora (1986), Kolb et al. (1986), Lines and Treitel (1984), Bamberger et al. (1982), for a small sample. All of the work

\footnotetext{
"Research supported in part by the Office of Naval Research under contract N000014-85K0725 and by the National Science Foundation under grant DMS86-03164.
} 
just cited is based on the output least-squares principle, according to which a subsurface model is required to generate (synthetic) sections which simultaneously fit a corresponding set of data sections in the least mean-square error sense. This approach does not require picked travel times, unlike reflection tomography (Bube et al. (1985), Lines et al. (1987)), and in principle could extract an optimal distribution of seismic velocities as well as reflection amplitudes, unlike linearized inversion (Ikelle et al. (1988), Cohen and Bleistein (1979), Clayton and Stolt (1981), Beylkin (1985)). In addition, any desired level of detail concerning the physics of seismic wave propagation may be built into the output-least-squares principle, and it may also incorporate non-seismic constraints (Lines et al. 1988).

In practice, it has proven difficult to realize the apparent promise of leastsquares inversion even when applied to synthetic data sets. Since the problems are computationally large, only iterative methods are feasible, and these require for their efficient convergence a measure of convexity often not possessed by the mean-square seismogram error (see e.g. Tarantola (1986), Hadjee and Collino (1988), Santosa and Symes (1986), (1987)).

The cause of this non-convexity is the extreme sensitivity of the synthetic seismogram to changes in the slowly-varying components of velocity, as will be reviewed in Section 2. Thus velocity trends emerge slowly or not at all as iteration proceeds, and the reflectivities are correspondingly degraded. For layered medium problems, Kolb and others have developed a number of continuation and reparameterization strategies which render the optimization more tractable (Kolb, Collino and Lailly (1986), Canadas and Kolb (1986)). Only limited tests of these devices (and no theoretical justification) have been reported, however, and it is difficult to understand how lateral heterogeneity might be accommodated by these techniques. Straightforward least-squares inversion of two-dimensional acoustic and elastic models has appeared to require a priori information of the gross model (velocity) features of very high quality and, when such information is provided, the results closely resemble those of carefully designed amplitude-preserving migration-unsurprisingly, as these are essentially equivalent (Lailly (1984), Gauthier et al. (1986), Mora (1987)). In fact, the principal tangible result of the work on least-squares inversion so far has been to provide a version of migration which may conceivably yield estimates of elastic reflection amplitudes (Tarantola (1984), Mora (1987), Burridge and Beylkin (1988)), hence a rational basis for amplitude versus offset analysis. While this is an important step, it appears that least-squares inversion has not so far advanced the estimation of velocities in regions of complex structure, and without such velocity information its depth-migration function is disabled as well (Santosa and Symes (1986), Spratt (1987)).

It is important to understand that the difficulty is not due to inadequate modeling of seismic wave propagation, or to the features of field data (beyond 
specifying the general character of any useful model). That is, least-squares inversion fails for essentially mathematical, rather than (geo-)physical, reasons. These reasons are explored in depth in the monograph, Santosa and Symes (1986).

The purpose of this paper is to propose another approach to full-waveform seismic reflection inversion, in which the coherence of multiple reflectivity estimates is optimized. While the basic ideas behind this approach are quite old, our use of them in designing an optimization algorithm for inversion seems new. Moreover, coherency optimization appears to avoid the mathematical pitfalls which often prove fatal to output-least-squares inversion while producing the same type of subsurface estimate.

Minimal requirements for a useful model-based inversion algorithm might be stated as follows:

(a) Stability: at least for data which are nearly model-consistent, the estimates of subsurface properties should "degrade gracefully" in the presence of data noise;

(b) Computability: for iterative methods, this means that convergence should occur at a reasonable rate, and for "poor" initial estimates, i.e., convergence should not require knowing the answer beforehand;

(c) Completeness: the algorithm should extract "most" of the information about the model implicit in the data, and the user should be able to have confidence that it has done so.

Of course, an algorithm which meets these conditions may still fail: besides these mathematical requirements, one must also impose the (geo-)physical requirement that the underlying model faithfully reflect the physics of the reflection seismology experiment, at least at the level of detail desired in the subsurface estimate. While this physical consistency is the ultimate limiting factor in the utility of any inversion algorithm, our focus in this paper will be limited to the necessary preconditions (a)-(c) above.

In the remainder of this introduction, we shall outline the coherency optimization algorithm in general terms. In succeeding sections we shall:

§2: formulate coherency optimization precisely for the simplest interesting model: layered, constant-density acoustics;

§3: analyse the algorithm of $\S 2$ in some detail, demonstrate that it possesses the attributes (a) through (c) above;

\$4: describe a numerical implementation, and exhibit the results of some numerical experiments, which establish feasibility; 
\$5: formulate coherency optimization for the simplest non-layered model, i.e. general constant-density acoustics, to demonstrate that this approach is not an artifact of wave propagation in layered media.

In the concluding Section 6 we recapitulate the properties of coherency optimization and its comparison with other approaches, and offer a few more observations concerning the range of models to which the technique might be applied.

The paper is written so that Sections 3 and 5 are not prerequisite to the others. The basic assertions of the paper are stated verbally in this introduction, then with some mathematical precision in Section 2, and illustrated numerically in Section 4. Thus the reader who wishes to avoid the analytical justification of these assertions can skip Section 3 without losing the thread of the argument, and those interested only in layered problems may likewise avoid Section 5.

Coherency optimization is easiest to describe in the context of the linearized seismogram, in which the model is regarded as the sum of smooth background model and a highly oscillatory ("rough") perturbation (reflectivity section). Note that the smooth background velocities are still regarded as part of the model, so it is still nonlinear. With some high-frequency approximations ("WKBJ Seismogram") the inversion of each common-source (or commonreceiver or common offset) gather for a given background velocity may be accomplished semi-analytically (perhaps the best reference is Beylkin (1985)), by what amounts to an amplitude-preserving before-stack migration. If the background model is correct, presumably the images from the various inverted gathers will line up. If not, the discrepancy indicates that the background ("migration") velocity ought to be changed.

So far, this is hardly novel: a process called "iterative before-stack migration" is described in just this way in Kleyn (1979), for instance. The refinement leading to a feasible algorithm is the introduction of a well-behaved cost function, which we dub the incoherence, which both measures the discrepancy between the various common gather inversions and indicates how the velocity fields should be changed to line them up (through its gradient). A natural choice is stack power or semblance (Fowler (1986), for example), but this leads directly back to the nonconvexity difficulties of output-least-squares: the two are very closely related (Santosa and Symes (1988), Appendix A). Instead, we depend on the accurate inversion of amplitudes, and take the mean-square of the differences between successive inverted gathers. These differences should all vanish at the "correct" velocity estimate. Most important, as the background velocity is changed, this incoherence (or "differential semblance") changes smoothly, rather than abruptly as is the case with stack power (this is illustrated in Section 4).

Two technical refinements are necessary to turn this idea into an algorithm. First, data noise which is uncorrelated from gather to gather will yield a very 
large contribution to the differences of the inverted gathers, completely distorting the incoherence and masking the correct choice of velocity. To avoid this oversensitivity to data noise, partly decouple the inverted gathers from the data: lump these reflectivities (one per gather) together with the background velocity field as the "model" to be determined, and redefine the cost to be the sum of:

(a) all of the mean-square data errors, i.e. differences between data seismogram and predicted seismogram based on the corresponding reflectivity section, and

(b) the mean-square sum of pairwise differences of reflectivity sections (incoherence).

Incoherent noise in the data gathers will be accounted for mostly in errorof-fit (i.e. (a)), as it should be, since it causes a much smaller increase in cost that way than when forced into the incoherence (i.e. (b)).

The second technical modification is required by the nature of the model-toseismogram relation: as pointed out above, this relation is extremely sensitive to the background velocity component, when the model is defined in terms of depth (and offset). This oversensitivity is due to the time shift accompanying a change in background velocity, which causes the temporal location of a highfrequency reflection from a (depth-) fixed reflector to change. For a discussion of this "time-shift" disaster in the one-dimensional context see Symes (1986). The remedy is clear: the reflectivity sections should be defined as time sections rather than as depth sections.

For the layered acoustic problem which occupies most of this paper, it is rather trivial to accomplish this transformation. It is particularly convenient to work with plane wave sources, since the gather corresponding to a planewave source at definite slowness has only one independent trace. Thus a single reflectivity time trace at each slowness, together with the (single) background velocity depth profile, specifies the model. The reflectivity time traces are converted to depth traces through the (background) vertical travel-time-to-depth transformation appropriate to each slowness. The high-frequency approximation to the ( $p$-tau) seismogram is simply the convolution of the time-trace reflectivities with the source wavelet whereas the incoherence is essentially the derivative of the corresponding depth-trace reflectivities with respect to slowness.

For laterally heterogeneous models, the transformation from time to depth is less obvious. As explained in Section 5, the analogue of the travel-time-to-depth change of variables appropriate to plane waves in layered media is in general a before-stack Kirchhoff migration appropriate to the acquisition geometry being simulated, and the reflectivity gather is simply a time section. The incoherence is a difference (or derivative) with respect to shot location (for shot gathers) of the migrated reflectivity depth sections. 
Two points remain to be emphasized. First, there is nothing to "cohere" unless reflectors are present, i.e. unless the reflectivity gathers are rich in highfrequency energy. As is the case with semblance, the incoherence is a measurement of the portion of moveout in reflection phase not accounted for by the current velocity model. Therefore the density of reflectors is a limiting factor in the recovery of accurate velocity models, as is the data aperture. These observations are quantified in Section 3.

Second, both incoherence and data misfit are summed in the cost functional for coherency optimization. Thus both are minimized simultaneously. The major theoretical result of the paper (Section 3) is that, with appropriate weights on the two components, sufficiently dense reflectors, and enough aperture the cost functional is smooth and convex for near-consistent data. The favorable computational consequences are exhibited in Section 4.

\section{Output least-squares vs. coherency optimiza- tion}

Denote the plane-wave ("p- $\tau$ ") seismogram corresponding to a velocity profile $c(z)$ by $S[c]$. The arguments in this paper are based on the well-known convolutional approximation, which is reasonably accurate when $c$ may be re-written as a sum

$$
c+\Delta c
$$

where

$c(z)$ is a slowly varying background velocity model

$\Delta c(z)$ is a rapidly varying "reflector sequence," having locally zero mean on the length scale of significant change in the background velocity.

Thus the (two-way) travel-time to depth $z$ of a precritical plane-wave at (horizontal) slowness $p$ is determined with a small error by the background velocity $c(z)$ according to

$$
\tau(z, p)=2 \int_{0}^{z} d z^{\prime}\left(\frac{1}{c^{2}(z)}-p^{2}\right)^{1 / 2}=2 \int_{0}^{z} \frac{1}{v}
$$

where $v$ is the vertical (plane-wave) velocity at slowness $p$ :

$$
\begin{aligned}
v(z, p) & =\left(\frac{1}{c^{2}(z)}-p^{2}\right)^{-1 / 2}=c(z) \Lambda(z, p) \\
\Lambda(z, p) & =\left(1-c^{2}(z) p^{2}\right)^{-1 / 2} .
\end{aligned}
$$


The convolutional approximation with (isotropic) source wavelet $f(t)$ is then

$$
\begin{aligned}
S[c, \Delta c](t, p) & =v(0, p)^{-1}\left(f * \frac{\partial r}{\partial t}\right)(t, p) \\
& =\frac{1}{v(0, p)} \int_{0}^{t} d t^{\prime} f\left(t-t^{\prime}\right) \frac{\partial r}{\partial t}\left(t^{\prime}, p\right)
\end{aligned}
$$

where the "reflectivity" $r(t, p)$ is given by

$$
r(t, p)=\frac{\Delta v(\zeta(t, p), p)}{v(\zeta(t, p), p)}
$$

by means of the inverse two-way travel-time function $\zeta$, defined implicitly by

$$
t=2 \int_{0}^{\zeta(t, p)} \frac{1}{v}
$$

and the vertical velocity perturbation

$$
\Delta v=\Delta c \cdot \Lambda^{3} .
$$

Note that reflectivity conventionally means $\partial r / \partial t$; we shall confuse $r$ and its $t$-derivative, calling both "reflectivity" as convenient.

Now $S$ is clearly linear in $\Delta c$, but quite nonlinear in $c$. In fact, a change in $c$ will typically result in a change in the "phase" $\zeta$, and thus in a shift in the high-frequency components of $S$, which in turn derive from the highfrequency components in $\Delta c$. Since such a phase-shift may have a drastic effect on components of the appropriate (high) frequency, and since $\Delta c$ must have a great deal of high-frequency content in order to model the dense distribution of reflectors in the typical sedimentary column, one expects $S$ to be extremely sensitive to changes in the background velocity $c$.

This oversensitivity to background errors shows quite clearly in the expression derived for the perturbation $\delta S$ in the seismogram, due to a perturbation $\delta c$ in the background velocity (holding $\Delta c$ fixed, and assuming $\delta c(0)=0$ ):

$$
\delta S(t, p)=v(0, p)^{-1} f * \frac{\partial}{\partial t}\left(-\frac{\Delta v}{v^{2}} \delta v+\frac{\partial}{\partial z}\left(\frac{\Delta v}{v}\right) \delta \tilde{\zeta}\right)(\zeta(t, p), p)
$$

where

$$
\begin{aligned}
& \delta \tilde{\zeta}(z, p):=\delta \zeta(\tau(z, p), p)= \\
& \quad v(z, p) \int_{0}^{z} d z^{\prime} v\left(z^{\prime}, p\right) c^{-3}\left(z^{\prime}\right) \delta c\left(z^{\prime}\right)
\end{aligned}
$$

is the phase perturbation corresponding to $\Delta c$, referred to depth/slowness coordinates. 
Thus the $z$-derivative of $\Delta c$ (or, of $r$ ) appears in the perturbations $\delta S$ associated with a background velocity perturbation $\delta c$. On the other hand, a perturbation $\delta \Delta c$ in $\Delta c$ simply results in

$$
\delta S \sim S[c, \delta \Delta c]
$$

as $S$ is linear in $\Delta c$ - thus no derivative of $\Delta c$ is involved.

Again, $\Delta c$ must be highly oscillatory to model the typical reflector distribution, so the derivative of $\Delta c$ is typically much bigger, in any reasonable sense, than is $\Delta c$ itself. Thus perturbation of the background velocity $c$ has a much larger effect on $S$ than does perturbation of $\Delta c$ : in the language of linear algebra, the linear perturbation map ("Jacobian")

$$
\delta c, \delta \Delta c \mapsto \delta S
$$

is ill-conditioned.

Even worse, a straighforward extension of this reasoning shows that the difference between the perturbed section

$$
S[c+\delta c, \Delta c+\delta \Delta c]
$$

and its linear prediction

$$
S[c, \Delta c]+\delta S
$$

can easily be on the order of $S$ itself, even for quite small $\delta c$.

To summarize: under realistic assumptions on $c$ and $\Delta c$,

(i) the Jacobian $\delta S$ is ill-conditioned;

(ii) $S$ is poorly approximated by its linearization.

Consequently, the output least-squares objective function

$$
J\left[c, \Delta c ; S_{d a t a}\right]:=\iint d p d t\left|S[c, \Delta c]-S_{d a t a}\right|^{2}
$$

is highly non-convex, with rapidly changing gradient, and the optimization problem

$$
\min _{c, \Delta c} J\left[c, \Delta c ; S_{d a t a}\right]
$$

is extremely difficult to solve by means of any variant of Newton's method. For extensive discussion and illustration of these points, consult Santosa and Symes [1986].

The crux of the difficulty is the interaction between the background $c$ and the reflectivity $r$ through the definition (2). Accordingly, it is tempting to decouple 
$c$ and $r$ by treating $r$, rather than $\Delta c$, as the "other" component of the model: thus define

$$
\tilde{S}[c, r](t, p)=v(0, p)^{-1} f * \frac{\partial r}{\partial t}(t, p) .
$$

Certainly if $r$ and $\Delta c$ are related by (2) then

$$
S[c, \Delta c]=\tilde{S}[c, r] .
$$

In fact, apart from the surface normalization, the background velocity $c$ enters the definition of $\tilde{S}$ only implicitly, through the condition (2). If we are to use $r$ as one of the independent variables, instead of $\Delta c$, we must develop a condition, phrased only in terms of $c$ and $r$, which guarantees that (2) holds. Fortunately, this is rather easy: from the useful identity

$$
\frac{\Delta v}{v^{3}} \equiv \frac{\Delta c}{c^{3}}
$$

it follows that, if (2) is satisfied, then

$$
c^{-3}(z) \Delta c(z)=v^{-2}(z, p) r(\tau(z, p), p) .
$$

The notable quality of (5) is that the left-hand side is independent of $p$. Thus differentiation with respect to $p$ eliminates $\Delta c$ altogether:

$$
0=\frac{\partial}{\partial p}\left[v^{-2}(z, p) r(\tau(z, p), p)\right] .
$$

It is easy to reverse this reasoning. Thus:

A section $r(t, p)$ is the reflectivity corresponding to $\Delta c(z)$ if and only if $(6)$ is satisfied, in which case $\Delta c$ is given in terms of $r(t, p)$ and $c(z)$ by (5).

In formulating the constraint given by (6), it is advantageous to return to $(t, p)$ coordinates (the reason will become apparent below). Thus define the quantity $C[c, r]$ (the incoherency) by

$$
C[c, r](t, p)=\left.\left\{\frac{\partial}{\partial p}\left[v^{-2}(z, p) r(\tau(z, p), p)\right]\right\}\right|_{z=\zeta(t, p)} .
$$

Then

$$
S[c, \Delta c]=S_{\text {data }}
$$

if and only if

$$
\begin{aligned}
\tilde{S}[c, r] & =S_{\text {data }} \\
C[c, r] & \equiv 0
\end{aligned}
$$


with $\Delta c$ and $r$ related by (2) or (5).

Since the problem is clearly overdetermined, it is commonplace to replace the exact fit of predicted to measured seismograms by a best-fit condition. We shall folow the lead of most authors on this subject (e.g. Tarantola and Vallette (1982)) and use the mean-square error measure. Some notation for this is desirable: for a single $z$-or $t$-trace, e.g. $f(t)$, we write

$$
\|f\|=\left(\int_{0}^{t_{\max }}|f(t)|^{2}\right)^{1 / 2}
$$

whereas for a $(t, p)$ or $(z, p)$ section, e.g. $F(t, p)$, we write

$$
\|F\|=\left(\int_{0}^{t_{\max }} d t \int_{0}^{p_{\max }} d p p|F(t, p)|^{2}\right)^{1 / 2} .
$$

The weight $p$ in the integral defining |||||| is a residue of polar coordinates, and is used to make the norm $\||\||$ as close as possible to equivalent to the ordinary mean-square of an $x-t$ section. See Santosa and Symes (1988), Appendix B; also Brysk (1986).

In view of the equivalence noted above, it is natural to pose the constrained least-squares problem:

$$
\begin{array}{ll}
\underset{c, r}{\operatorname{minimize}} & \left\|\tilde{S}[c, r]-S_{\text {data }}\right\| \|^{2} \\
\text { subject to } & C[c, r] \equiv 0 .
\end{array}
$$

This problem is closely related to the output least-squares problem (3). In fact, the relation is too close. The two problems have the same solution, and in fact are mathematically quite similar as well, so that (7a) is also poorly suited to computation. To obtain a problem which is not too "stiff" we "relax" (7a) by making the constraint $C=0$ soft:

$$
\underset{c, r}{\operatorname{minimize}} \tilde{J}\left[c, r, S_{\text {data }}\right]
$$

where

$$
\tilde{J}\left[c, r, S_{\text {data }}\right]=\left\|\tilde{S}[c, r]-S_{\text {data }}\right\|\left\|^{2}+\sigma^{2}\right\| C[c, r] \|\left.\right|^{2} .
$$

We shall call (7b) the coherency optimization problem.

From the equivalence above, we see that if $S_{\text {data }}$ is consistent, i.e. $S_{\text {data }}=$ $S[c, \Delta c]$, then the problem (7b) has $[c, r]$ amongst its solutions for which $r$ and $\Delta c$ are related by (5). That is, for consistent data, (7b) has "the same solutions" as the output least-squares problem (3). We shall show, however, that (7b) is far better suited to numerical computation by Newton's method and its relatives. 
We shall also show that the solution of $(7 \mathrm{~b})$ is stable, i.e. "degrades gracefully" in the presence of data error, for reasonable choices of the penalty parameter $\sigma$.

These conclusions are true provided that $S_{\text {data }}$ is near (in the mean-square sense) some "exact" or consistent data $S[c, \Delta c]$, and provided that $c, \Delta c$ satisfy certain conditions. "Physical," or poetic, statements of these conditions are:

(i) $\Delta c$ should be "rough", i.e. contain significant variation (reflectors), on a length scale dictated both by the wavelet $(f)$ passband and by the smoothness (characteristic length) of $c$;

(ii) The range of slownesses $p$ available in the data ("aperture") should be sufficiently large relative to both the degree of roughness mentioned in (i) and the amount of data error, so that the moveout of reflections clearly discriminates the velocities.

It is also necessary that the penalty parameter $\sigma$ be chosen appropriately. "Appropriate" means, so that the desired properties are obtained, i.e., so that Newton's method works (from a poor initial guess) and so that the solution obtained is relatively insensitive to data error. The main theoretical result of this paper is that such an appropriate choice of $\sigma$ is possible, provided that (i) and (ii) are satisfied in a suitable sense, and that this choice is robust.

Note that in the limit $\sigma \rightarrow \infty$, (7b) becomes (7a), i.e. the constraint becomes hard. Previous remarks have indicated that (7a) (or (3)) is not susceptible to numerical solution through Newton's method or its relatives. Thus the possibility of choosing $\sigma$ "not too large" is crucial to the construction of algorithms which converge from a poor initial guess.

In the following section we will carefully quantify (i) and (ii) and justify these conclusions through an analysis of $(7 \mathrm{~b})$, and in section 4 we will present the results of some numerical experiments, along with a discussion of implementation issues (e.g. choice of $\sigma$ ).

\section{Analysis of the coherency optimization prob- lem}

The local analysis of optimization problems amounts to the verification of several conditions concerning the first and second derivatives of the objective function. These conditions are imposed near a particular solution, and guarantee that this solution is stable under data perturbations. The same conditions ensure rapid local convergence of Newton's method. We state the verbal description of these conditions here, together with the interpretation in terms of the quantities introduced in the last section: 
(i) (Regularity) The objective function should be twice differentiable near the solution.

This is completely obvious for the seismogram error, as it is independent of $c$ and quadratic in $r$. For the incoherency, the regularity is obvious from the identity

$$
\begin{aligned}
C[c, r](t, p) & =\left.\frac{\partial}{\partial p}\left(v(z, p)^{-2} r(\tau(z, p), p)\right)\right|_{z=\zeta(t, p)} \\
& =-2 p r(t, p)+v^{-2}(\zeta(t, p), p)\left\{\frac{\partial r}{\partial p}(t, p)-p \frac{\partial r}{\partial t}(t, p) \int_{0}^{\zeta(t, p)} v\right\}
\end{aligned}
$$

Clearly varying $c$ will have the effect of differentiating $v$ - but $c$, hence $v$, is presumed sufficiently smooth that its derivative is not significantly larger than $c$ itself. On the other hand, no additional derivatives of $r$ (the locus of highfrequency energy) result from varying $c$. Thus $C$ may be regarded as regular. Note that the perturbation of $C$ would involve further derivatives of $r$ if we had left off the final referral back to time/slowness coordinates.

(ii) (second-order sufficiency): The objective Hessian is positive-definite.

Most of this section is devoted to verifying this last condition; it is the source of the requirements mentioned at the end of the last section.

The Hessian is the second-order coefficient $\delta^{2} \tilde{J}$ in the power series expansion

$$
\begin{aligned}
& \tilde{J}\left[c+\epsilon \delta c, r+\epsilon \delta r, S_{d a t a}\right] \\
& \quad=\tilde{J}\left[c, r, S_{\text {data }}\right]+\epsilon \delta \tilde{J}+\frac{\epsilon^{2}}{2} \delta^{2} \tilde{J}+\ldots
\end{aligned}
$$

In view of the goal, enunciated at the end of the last section, to examine the perturbation of a consistent solution due to perturbing the data, we shall assume that

$$
\tilde{J}\left[c, r, S_{\text {data }}\right]=0 .
$$

That is, that

$$
\tilde{S}[c, r]=S_{\text {data }} \text { and } C[c, r]=0 .
$$

Let us state very carefully the meaning of the condition (ii) in terms of $\delta^{2} \tilde{J}$ : i.e. $\delta^{2} \tilde{J}$ is positive. Condition (ii) actually requires that $\delta^{2} J$ be "positive relative to" $\delta c, \delta r$ : for some $L>0$,

$$
\|\| \delta^{2} \tilde{J} \| \mid \geq L\left(\|\delta c\|^{2}+\left.\||| \delta r\|\right|^{2}\right) .
$$

Indeed, as follows from the proof of the implicit function theorem, as $L \rightarrow 0$ in (10), the size of the region of convexity (within constrained models) of $\tilde{J}$ goes to 
zero, and the possible ratio of solution error to data error goes to infinity. Thus we need not only $L>0$, but some positive control over $L$, to ensure stability (and computability) of the solution.

An easy calculation gives

$$
\delta^{2} \tilde{J}=2\|\| f * \frac{\partial}{\partial t} \delta r\|\|^{2}+2 \sigma^{2}\|\| \delta C\|\|^{2} .
$$

Thus as a first requirement, we must have (for each $p$ )

$$
\left\|f * \frac{\partial}{\partial t} \delta r\right\| \geq K_{1}\left\|\frac{\partial}{\partial t} \delta r\right\|
$$

for some constant $K_{1}>0$. Unless $K_{1}$ is to be uselessly tiny, this means in effect that each trace $\delta r(p=$ const.) (hence eventually $r$ ) must have most of its energy in the passband of the source $f$, or else that the out-of-band components must be constrained a priori. We adopt the first option: i.e., that we shall estimate only passband reflectivities. In quantitative terms, $K_{1}$ should be at least some substantial fraction, say one-half, of the absolute moment of the wavelet (this choice is justified by the theory of convolution operators, and is essentially optimal):

$$
K_{1} \approx .5 \int|f|
$$

Inequality (11) only shows that $\delta^{2} \tilde{J}$ majorizes $\left.\left\|\frac{\partial \delta r}{\partial t}\right\|\right|^{2}$. Reference to (10) shows that this is inadequate-unsurprisingly, since we have not made full use of the hypotheses of (ii). To go further, we must introducd the linearized constraints (since otherwise $\delta c$ is unconstrained). As we have assumed $c, r, S_{\text {data }}$ consistent, i.e. the system of equations (9), this simplifies considerably. The calculation is displayed in the Appendix: with $\Delta c$ given by (5), we obtain the identities

$$
\begin{aligned}
\delta C= & \delta_{c} C+\delta_{r} C \\
\delta_{c} C= & -2 p\left\{2\left[(Q \delta c) \Lambda c^{-1}+\left(\int_{0}^{z} Q \delta c\right) p^{2} \Lambda^{3} c^{\prime}\right]\left(\frac{\Delta c}{c}\right)\right. \\
& \left.\quad+\left[\left(\int_{0}^{z} Q \delta c\right) c^{-1} \Lambda\right] \frac{\partial}{\partial z}\left(\frac{\Delta c}{c}\right)\right\}\left.\right|_{z=\zeta} \\
\delta_{r} C= & C[c, \delta r] \\
= & \left\{\frac{\partial}{\partial p}\left(v^{-2} \delta r \circ \tau\right)\right\} \circ \zeta
\end{aligned}
$$

Here the quantity $Q \delta c$ is defined by

$$
\begin{aligned}
Q \delta c: & =[\delta(v \circ \zeta)] \circ \tau \\
& =\Lambda^{3}\left(\delta c+c^{\prime} v \int_{0}^{z} v \frac{\delta c}{c^{3}}\right) .
\end{aligned}
$$


Also, we have used the useful notation

$$
g \circ \tau(z, p)=g(\tau(z, p), p)
$$

for (any) function $g(t, p)$ and similarly for the symbol o $\zeta$.

The further analysis of this condition in general is a little involved. We consider in detail only the special case

$$
\begin{array}{cl}
c & \text { (hence } v \text { ) constant for } z \geq z_{0} \\
\delta c & \text { (hence } \delta v) \equiv 0 \text { for } z \leq z_{0}
\end{array}
$$

A few remarks about the general case appear near the end of this section. See Figure 1.

Under this restriction, $\delta_{c} C$ simplifies considerably: $Q \delta c=\Lambda^{3} \delta c$, and

$$
\delta_{c} C \circ \tau=-2 p \Lambda^{4} c^{-2}\left\{2 \delta c \Delta c+\left(\int_{0}^{z d z} \frac{\partial \Delta c}{\partial z}\right)\right\} \text {. }
$$

Now

$$
\frac{\partial}{\partial p} \Lambda^{2}=2 p c^{2} \Lambda^{4} .
$$

Thus what remains of (12a) is an exact derivative in $p$, so we integrate in $p$ from $p_{1}$ to $p_{2}$ to obtain

$$
\begin{gathered}
\left.c^{-4}\left\{2 \delta c \Delta c+\left(\int_{0}^{z} \delta c\right)\left(\frac{\partial \Delta c}{\partial z}\right)\right\} \Lambda^{2}\right|_{p_{2}} ^{p_{1}}=\left.v^{-2}(\delta r \circ \tau)\right|_{p_{1}} ^{p_{2}}+\int_{p_{1}}^{p_{2}} d p\left(\delta_{q} C \circ \tau\right) \\
=\left.c^{-2} \Lambda^{-2}(\delta r \circ \tau)\right|_{p_{1}} ^{p_{2}}+\int_{p_{1}}^{p_{2}} d p\left(\delta_{q} C \circ \tau\right) .
\end{gathered}
$$

Equation (14) will be the "hook" to get $\delta^{2} \tilde{J}$ into an explicit relation with $\delta c$. This is accomplished in three steps. First, we make use of an important by-product of the assumed bandlimited feature of $f$ :

Since $f$, hence $r, \delta r$, has its energy concentrated away from 0 Hertz, the t-derivative of $\delta r$ is at least as big (probably much larger) than $\delta r$ itself: for each $p$,

$$
K_{2}\|\delta r\|^{2} \leq\left\|\frac{\partial \delta r}{\partial t}\right\|^{2}
$$

for a constant $K_{2}$ depending on the lowest passband frequency.

Together with (11), this gives

$$
K_{2} K_{1}^{2}\|\delta r\| \|^{2} \leq \delta^{2} \tilde{J} .
$$


The second step is to manipulate (14) into an inequality with $\|\mid \delta r\| \|^{2}$ on the right-hand side. This is accomplished with the aid of several identities relating time and depth, which are massively simplified by assuming that $c \equiv$ const. where $\delta c \neq 0$, as we have done.

According to the hypothesis (13), for $z>z_{0}$

$$
\begin{aligned}
c(z) & =c_{1} \quad \text { for } z>z_{0} \\
\tau(z, p) & =\tau_{0}(p)+\frac{2\left(z-z_{0}\right)}{c_{1} \Lambda_{1}(p)}
\end{aligned}
$$

where

$$
\tau_{0}(p)=\tau\left(z_{0}, p\right)
$$

and

$$
\Lambda_{1}(p)=\left(1-c_{1}^{2} p^{2}\right)^{-1 / 2}
$$

Thus (since (14) implies $\delta r \circ \tau=0, z<z_{0}$ ):

$$
\begin{aligned}
\|\delta r \circ \tau\|^{2} & =\int d z|\delta r(\tau(z, p), p)|^{2} \\
& =\frac{1}{2} c_{1} \Lambda_{1}(p) \int d t|\delta r(t, p)|^{2} \\
& =\frac{1}{2} c_{1} \Lambda_{1}(p)\|\delta r\|^{2} .
\end{aligned}
$$

Now set $p_{1}=p, p_{2}=\frac{1}{2} p_{\max }+p$, and integrate $\sqrt{p}$ times the first summand on the r.h.s. of (14) from $p=0$ to $p=\frac{1}{2} p_{\max }$ (for fixed $z$ ) neglecting the factor of $c^{-2}$ :

$$
\begin{aligned}
& \pm\left.\int_{0}^{\frac{1}{2} p_{\max }} d p \sqrt{p}\left(\Lambda_{1}^{-2} \delta r \circ \tau\right)\right|_{p} ^{p+\frac{1}{2} p_{\max }} \\
& \leq \int_{0}^{\frac{1}{2} p_{\max }} d p \sqrt{p}\left\{\left.\Lambda_{1}^{-2}|\delta r \circ \tau|\right|_{p+\frac{1}{2} p_{\max }}+\Lambda_{1}^{-2}|\delta r \circ \tau|_{p}\right\} \\
& \leq \int_{0}^{p_{\max }} d p \sqrt{p} \Lambda_{1}^{-2}|\delta r \circ \tau| \\
& \leq\left(\int_{0}^{p_{\max }} d p \Lambda_{1}^{-3}\right)^{\frac{1}{2}}\left(\int_{0}^{p_{\max }} d p p \Lambda_{1}^{-1}|\delta r \circ \tau|^{2}\right)^{\frac{1}{2}} .
\end{aligned}
$$

Applying the same step to the absolute value of the l.h.s. of (14) gives

$$
c_{1}^{-4}\left\{2 \delta c \Delta c+\left(\int_{0}^{z} \delta c\right)\left(\frac{\partial \Delta c}{\partial z}\right)\right\} \lambda\left(c_{1}, p_{\max }\right)
$$

where

$$
\lambda\left(c_{1}, p_{\max }\right):=\int_{0}^{\frac{1}{2} p_{\max }} d p \sqrt{p}\left(\Lambda_{1}^{2}\left(p+\frac{1}{2} p_{\max }\right)-\Lambda_{1}^{2}(p)\right) .
$$

The modulus $\lambda\left(c_{1}, p_{\max }\right)$ satisfies $\lambda(0)=0$ and $\lambda\left(c_{1}, p_{\max }\right) \rightarrow \infty$ as $p_{\max } \rightarrow 1 / c_{1}$ (since the integrand has a nonintegrable singularity at $p_{\max }=1 / c_{1}$ ). Otherwise $\lambda$ is best investigated numerically; a plot of $\lambda$ vs. $c_{1}, p_{\max }$ appears as Figure 2. 
Clearly, in order that $\lambda \gg 0$ it is necessary that $c_{1} p_{\max }$ be relatively close to 1.

Since (17) is valid with either sign, we can square both sides of the inequality we have just derived from (14), (17), (18) and make an obvious estimate of the second term on the r.h.s. of (14) to get:

$$
\begin{aligned}
& \left\{2 \delta c \Delta c+\left(\int_{0}^{z} \delta c\right)\left(\frac{\partial \Delta c}{\partial z}\right)\right\} \\
\leq & \lambda\left(c_{1}, p_{\max }\right)^{-2} c_{1}^{2}\left[\left(\int_{0}^{p_{\max }} d p \Lambda_{1}^{-3}\right) \int_{0}^{p_{\max }} d p p \Lambda_{1}^{-1}|\delta r \circ \tau|^{2}\right. \\
& \left.+\frac{1}{4} c_{1}^{2} p_{\max }^{3} \int_{0}^{p_{\max }} d p p|\delta C \circ \tau|^{2}\right],
\end{aligned}
$$

integrate both sides in $z$, and make use of (16):

$$
\begin{aligned}
& \left\|2 \delta c \Delta c+\left(\int_{0}^{z} \delta c\right)\left(\frac{\partial \Delta c}{\partial z}\right)\right\|^{2} \\
& \leq\left.\lambda_{1}\left(c_{1}, p_{\max }\right)\|\delta r\|\right|^{2}+\lambda_{2}\left(c_{1}, p_{\max }\right)\|\delta \not d\|^{2}
\end{aligned}
$$

where $\lambda_{1}$ is given by

$$
\lambda_{1}\left(c_{1}, p_{\max }\right)=\frac{1}{2} c_{1}^{3} \lambda\left(c_{1}, p_{\max }\right)^{-2} \int_{0}^{p_{\max }} \Lambda_{1}^{-3}
$$

and

$$
\lambda_{2}\left(c_{1}, p_{\max }\right)=\frac{1}{4} c_{1}^{5} \lambda\left(c_{1}, p_{\max }\right)^{-2} \int_{0}^{p_{\max }} \Lambda_{1}^{-3} .
$$

A plot of $\lambda_{1}$ appears as Figure 3. Evidently:

In order that $\lambda_{1}$ be small, it is necessary that $c_{1} p_{\max }$ be relatively large (near 1).

This is still not quite what is required, as we have an estimate on the left-hand side of (18), not on $\delta c$ itself. The third step amounts to the observation that, if $\delta c$ is smooth enough, and $\Delta c$ rough enough, then this quantity cannot be small without $\delta c$ itself being small.

This observation is the most physically-meaningful, and the most difficult to quantify, of the points presented in this paper. As illustrated thoroughly in Santosa and Symes (1986), the local behaviour of the seismogram function near a rough model is quite different from that near a smooth model, and it is this property that is being used implicitly at this point.

A rigorous semiquantitative treatment of roughness is found in Symes (1988), and the present problem can be treated along the same lines. Such rigorous argument is neither particularly enlightening, however, nor very precise. For the present, a numerical illustration of this property will suffice. The smoothness 
of the velocity model $c$ and its perturbation are guaranteed for example by insisting that

$$
\begin{aligned}
c(z) & =c_{0} \exp \left\{\sum_{j=1}^{N} x_{j} \psi_{j}(z)\right\} \\
\delta c(z) & =\sum_{j=1}^{N} \delta x_{j} \psi_{j}(z) \cdot c(z)
\end{aligned}
$$

where $\left\{\psi_{j}\right\}$ is a finite, smooth set of basis functions. In the tests reported in Section 4 , cubic $b$-spline integrals are selected for the $\left\{\psi_{j}\right\}$ : these have associated with them a definite length scale ("width"); see Figure 4 . Then in vector notation,

$$
\|\delta c \Delta c\|^{2}=\delta x^{T} R^{0} \delta x
$$

where

$$
R_{i j}^{(0)}=\int d z \psi_{i}(z) \psi_{j}(z) c^{2}(z) \Delta c^{2}(z)
$$

is the $N \times N$ symmetric positive-semidefinite "roughness matrix" associated with $\Delta c$ and the basis $\left\{\psi_{j}\right\}$.

Similarly

$$
\|\delta c\|^{2}=\delta x^{T} M \delta x
$$

and

$$
\left\|\left(\int_{0}^{z} \delta c\right) \frac{\partial \Delta c}{\partial z}\right\|^{2}=\delta x^{T} R^{(1)} \delta x
$$

where

$$
R_{i j}^{(1)}=\int d z\left(\int_{0}^{z} \psi_{i}\right)\left(\int_{0}^{z} \psi_{j}\right) c^{2}(z)\left(\frac{\partial \Delta c}{\partial z}(z)\right)^{2}
$$

and $M$ is the symmetric positive-definite "mass matrix"

$$
M_{i j}=\int d z \psi_{i}(z) \psi_{j}(z) c^{2}(z)
$$

It follows that

$$
\mu_{\max }^{(0)}\|\delta c\|^{2} \geq\|\delta c \Delta c\|^{2}
$$

where $\mu_{\max }^{(0)} \geq 0$ is the largest eigenvalue of the $N$-dimensional generalized eigenvalue problem

$$
R^{(0)} y=\mu M y
$$

Likewise,

$$
\mu_{\min }^{(1)}\|\delta c\|^{2} \leq\left\|\left(\int_{0}^{z} \delta c\right) \frac{\partial \Delta c}{\partial z}\right\|^{2}
$$

where $\mu_{\min }^{(1)}$ is the least eigenvalue of the generalized eigenvalue problem

$$
R^{(1)} y=\mu M y
$$


Finally,

$$
\mu_{\min }\|\delta c\|^{2} \leq\left\|2 \delta c \Delta c+\left(\int_{0}^{z} \delta z\right) \frac{\partial \Delta c}{\partial z}\right\|^{2}
$$

where $\mu$ is the least eigenvalue of the generalized eigenvalue problem

$$
R y:=\left(2 R^{(0)}+R^{(1)}\right) y=\mu M y .
$$

Combining (19), (20a), (20c) and (20d) we get

$$
\mu_{\min }\|\delta c\|^{2} \leq \lambda_{1}\left(c_{1}, p_{\max }\right)\|\delta r\|\left\|^{2}+\lambda_{2}\left(c_{1}, p_{\max }\right)\right\| \delta \delta C \|^{2}
$$

and

$$
\mu_{\min } \geq\left(\mu_{\min }^{(1)}-4 \mu_{\max }^{(0)}\right) .
$$

Obviously inequality (21b) has force only if $\mu_{\min }^{(1)}>>\mu_{\max }^{(0)}$, that is, $\partial \Delta c / \partial z$ is much bigger than $\Delta c$, whence $\Delta c$ must be rough. In fact, $\Delta c$ must be uniformly rough on the length scale of significant variation in $c$. To see this, note that the worst possible situation is when $R^{(0)}$ and $R^{(1)}$ have a common null vector, since then the left-hand side of (19) constrains the corresponding component not at all. This can indeed occur. Note that the integrated spline basis $\left\{\psi_{j}\right\}$ is so constructed that $\psi_{j}-\psi_{j+1}$ vanishes outside an inteval $I_{j}$, encompassing five spline nodes: see Figure 5. Suppose that for some $j, \Delta c \equiv 0$ in the interval $I_{j}$ : that is, there are no reflectors in $I_{j}$. Set $\delta x_{j}=1, \delta x_{j+1}=-1, \delta x_{i}=0$ for $i \neq j$. Then $R^{(j)} \delta x=0, j=0,1$ so $\mu=0$ with eigenvector $\delta x$ for both matrices. Thus:

$$
\begin{aligned}
& \text { In order that } \mu_{\min }^{(1)}>4 \mu_{\max }^{(0)}>0 \text {, significant reflectors must be } \\
& \text { present in every depth interval of the characteristic length scale } \\
& \text { of the smooth model class. }
\end{aligned}
$$

In fact, it turns out that this condition is also necessary in order that the "combined" eigenvalue $\mu_{\min }$ be reasonably large, as we shall see below.

Note also the connection with the wavelet passband. In order for the convolutional model to be accurate, $c$ must have almost all of its energy concentrated below the passband (or rather its spatial analogue): that is, $c$ must be smooth on the spatial wavelength scale. Suppose that $c$ is chosen to attain, roughly, the maximum degrees of freedom permitted by this constraint. Thus the length scale associated with the background model is roughly the largest spatial wavelength in the data, and we can re-phrase the above conclusion as:

In order that (19) above constrain $\delta c$, significant reflectors must be present in every depth interval longer than the longest spatial wavelength in the data. 
This necessary condition is actually also sufficient, when made appropriately precise (Symes 1988; see also Santosa and Symes 1986). We illustrate the sufficiency by introducing the reflector sequence $\Delta c$ figuring in the experiments of Section 4. See Figure 6. We have computed the extreme eigenvalues of the problems, $(20 \mathrm{~b}),(20 \mathrm{~d}),(20 \mathrm{e})$. For $N$-dimensional integrated spline space, the characteristic length scale (i.e. the length of $I_{j}$ ) is proportional to $1 / N$. In Figure 7 , we tabulate $\mu_{\max }^{(0)}, \mu_{\min }^{(1)}$, and $\mu_{\min }$ against $N$, along with the characteristic length scale; for the background velocity profile we have taken the profile in Figure 6 also. We have not restricted the non-zeros of $\delta c$ to the $c=$ const. segment, so this is a more severe test than warranted by the present discussion. Evidently, the criterion above is indeed sufficient to guarantee $\mu_{\min }^{(1)}-4 \mu_{\max }^{(0)}>0$ in this case and appears necessary to have $\mu_{\min }>0$.

We now combine the conclusions of three steps: (13), (19), (20e), to get

$$
\frac{\mu_{\min }}{\gamma}\|\delta c\|^{2} \leq \delta^{2} \tilde{J}, \quad \gamma=\frac{\lambda_{1}}{K_{2} K_{1}^{2}}+\frac{\lambda_{2}}{\sigma^{2}}
$$

and, using (15) again

$$
\min \left(\frac{1}{2} K_{2} K_{1}^{2}, \frac{\mu_{\min }}{\gamma}\right)\left(\|\delta c\|^{2}+\|\| \delta r \|^{2}\right) \leq \delta^{2} \tilde{J}
$$

which gives an explicit estimate for the constant $L$ in (10), as required. Note that this constant of proportionality is neatly separated into $\mu_{\min }$, which depends only on the roughness of $\Delta c$, relative to $\delta c$, and $\gamma$, which depends on aperture and passband amplitude, and on the penalty parameter $\sigma$.

Note the role of $\sigma$ in (22): it is only the quotient $\lambda_{2} / \sigma^{2}$ which influences the lower bound. The aperture-dependent $\lambda_{1}$ and $\lambda_{2}$ are of roughly the same size, so the requirement that the lower bound be reasonable imposes a (rather loose) relation between the aperture, the passband amplitudes $\left(K_{1}, K_{2}\right)$, and $\sigma$ : in effect, a (soft) floor is placed under $\sigma$. On the other hand, the upper limit of the spectrum of $\delta^{2} J$ clearly grows with $\sigma^{2}$. Thus for "moderate" values of $\sigma$, the $\delta^{2} J$ is as well-conditioned as possible. Several possibilities exist for pinning down this range: guessing (the option pursued in numerical work reported here), rigorous theoretical estimation (far too conservative), or adaptive estimation during iterative solution of the linear stage of Newton's method (a project for the future).

Recall that the argument leading to this conclusion was based on the restriction (13). Without this restriction, the argument becomes more involved, but the conclusion is qualitatively the same: for sufficiently rough $\Delta c$, an inequality like (22) holds. It is evident from the form of the expression $Q \delta c$ that $c^{\prime} \neq 0$ will degrade the contribution of large $-p$ traces to the lower bound, and this is indeed the main quantitative effect. A rigorous argument of this nature, in the context of the fully nonlinear problem, can be found in Symes (1988). 


\section{Numerical Experiments}

In this section we report an implementation of the algorithm suggested in Section 2, and the results of some numerical experiments which establish its feasibility. More extensive tests of the method will be reported elsewhere.

The implementation requires (1) a choice of discretization of the parameter space and operators; (2) a choice of optimization strategy. We give sufficient detail in both areas that the interested reader should be able to reproduce our results.

\subsection{Discretization}

Both to maintain applicability of the convolutional model, and for the more subtle reasons given in Section 3, it is necessary that the background velocity $c$ be quite smooth-much smoother than the reflectivity, for example. A simple explicit way (though certainly not the only way) to ensure a given degree of smoothness is to choose $c$ from a finite-dimensional function space spanned by smooth functions.

For the space velocity profiles we took a manifold of exponentiated integrated $b$-splines (see Figure 4):

$$
c(z)=c_{0} \exp \left\{\int_{0}^{z} d z^{\prime} \sum_{i=1}^{n m o d} x_{i} \psi_{i}\left(z^{\prime}\right)\right\}
$$

where $\psi_{i}(z)=\Psi\left(n s p l \cdot\left(\frac{z-z_{i}+1}{z s p l}\right)\right), \Psi$ a standard $b$-spline, and $z_{i}=\frac{i}{n s p l} z s p l$, $i=0, \ldots, n s p l$ are $n s p l+1$ evenly spaced nodes. We set $n \bmod =n s p l-3$, so that all summands vanish at $z=0$ and $z=z s p l$. See Figure 4 .

The surface velocity $c_{0}$ was regarded as a fixed parameter.

The perturbations $\delta c$ have the form

$$
\delta c(z)=\left(\sum_{i=1}^{n m o d} \delta x_{i} \int_{0}^{z} \psi_{i}\right) c(z) .
$$

When values $z>z s p l$ are needed, both $c$ and $\delta c$ are regarded as constant and equal to their values at $z s p l$.

The depth functions occurring in the various formulae are sampled on a fixed grid $\{j d z: 0 \leq j \leq n z\}$, where $n z \cdot d z=: z_{\max } \geq z s p l$ ("the $z$-grid"). Routines were written which convert sampled $z$-grid functions $(c, \delta c)$ into spline coefficients $(x, \delta x)$ and vice versa, and which satisfy certain adjointness conditions detailed below. 
Time-slowness sections are regarded from the outset as sampled on a fixed grid of size $(n t+1) \times n p$, at given sample intervals $d t$ and $d p$. We used one-way time as the time parameter throughout.

\subsection{Operators}

The formulas for the incoherency $C$ and its perturbation $\delta C$ from the appendix and Section 2 require the travel-time change-of-variable and its inverse. These were accomplished via interpolation. For example, the integral

$$
\tau(z, p)=\int_{0}^{z} \frac{d z^{\prime}}{v\left(z^{\prime}, p\right)}
$$

is approximated using the trapezoidal rule on the $z$-grid, yielding an unequally spaced set $\left\{\tau_{i}\right\}$ of travel-times. The time-function to be converted to a depth function must then be evaluated at the $\tau_{i}$, which is done via piecewise-linear interpolation. The total process has a truncation error on the order of $d z^{2}$.

Derivatives with respect to $z, t$ and $p$ are replaced by simple 3-point centered difference formulas, maintaining the truncation order. Discretization of $C$ and $\delta C$ was accomplished by means of the formulas indicated in the Appendix.

The synthetic $p$-tau seismogram $\tilde{S}[c, r]$ was computed using a centered 3 point $t$-difference and trapezoidal rule approximation of the convolution integral.

\subsection{Norms}

The definition of the cost function $\tilde{J}$ involves the $L^{2}$-norm. As noted in Section 2 , this should really include a factor of $p$, to most closely maintain the relation with mean-square error in $(x, t)$ domain. To simplify our calculations, however, we ignored this point and defined the section $L^{2}$ norms by

$$
\|\left.|F|\right|^{2}=\sum_{j=0}^{n t} \sum_{k=1}^{n p} d t d p w_{t}(j) w_{p}(k)\left|F_{j k}\right|^{2}
$$

where

$$
\begin{aligned}
& w_{t}(j)= \begin{cases}1 / 2 & \text { if } j=0, n t \\
1 & \text { else }\end{cases} \\
& w_{p}(k)= \begin{cases}1 / 2 & \text { if } k=1, n p \\
1 & \text { else }\end{cases}
\end{aligned}
$$

i.e. the trapezoidal rule. With this choice, $\tilde{J}$ is completely defined.

The importance of choice of norms in the model space $[c, r]$ cannot be overemphasized. Even though the definition of $\tilde{J}$ is independent of this choice, it is 
the main factor affecting the efficiency of the optimization. This is principally because of the role of the model norm in the definition of the gradient. We examine this point for the summand $\sigma^{2}\|C[c, r]\| \|^{2}$ of $\tilde{J}$, since it involves both $c$ and $r$. By definition, the gradient of this term is the (unique) model vector $[\dot{c}, \dot{r}]$ for which for any $(\delta c, \delta r)$,

$$
\begin{aligned}
\lim _{\epsilon \rightarrow 0} & \frac{1}{\epsilon}\left(\sigma^{2}|\|C[c,+\epsilon \delta c, r+\epsilon \delta r]\||^{2}-\sigma^{2}\|C[c, r]\| \|^{2}\right) \\
& =\langle(\dot{c}, \dot{r}),(\delta c, \delta r)\rangle_{M}
\end{aligned}
$$

where $<,>_{M}$ is the scalar product in model space corresponding to the norm:

$$
\|[\delta c, \delta r]\|_{M}^{2}=\langle[\delta c, \delta r],[\delta c, \delta r]\rangle_{M} .
$$

(We assume that model space is a Hilbert space, since all efficient smooth optimization methods are predicated on this assumption.)

A principal requirement ((i) in Section 3$)$ is that the function $[c, r] \rightarrow$ $\left\||C[c, r] \||^{2}\right.$ is regular, i.e., differentiable. Examining (A2), we see that derivatives of $\delta r$ are involved in $\delta C$. Since $r, \delta r$ are to be allowed to be arbitrary (grid-representable) functions, $\delta C$ can ony be continuously dependent on $\delta r$, as is required by regularity, if the model norm includes explicit control over derivatives of $\delta r$. The obvious choice for the section part of the model norm is

$$
\begin{aligned}
\|\delta r\|_{1}^{2}= & \sum_{j=0}^{n t} \sum_{k=1}^{n p} d t d p w_{t}(j) w_{p}(k)\left\{\left|\delta r_{j k}\right|^{2}\right. \\
& \left.+\left|D_{t} \delta r_{j k}\right|^{2}+\left|D_{p} \delta r_{j k}\right|^{2}\right\}
\end{aligned}
$$

where $D_{t}$ and $D_{p}$ are 2-point one-sided difference approximations for $\partial / \partial t$ and $\partial / \partial p$. The subscript " 1 " stands for "first derivatives"; this is the discrete version of the first in the Sobolev scale of norms, a basic tool in modern analysis of partial differential equations.

For the velocity profile part, i.e. $\delta c$, we can make use of the fact that $C$ is required to belong to a space of smooth splines.

We enforce the membership of $c$ in the spline manifold by parameterizing the model in terms of the spline coefficients $x_{i}$ themselves, rather than the $z$-grid values of $c$. Also, we tacitly use $\log c$ and its perturbation, $\delta c / c$, as fundamental quantities rather than $c$ and $\delta c$; this nondimensionalizes that part of the model (note that $r$ is already non-dimensional, by definition). Thus we need to express the norm of $\delta c / c$ in terms of the $\delta x_{i}$; this is easily accomplished via the mass matrix

$$
M_{i j}=\int d z \psi_{i} \psi_{j}
$$

This is computed via the trapezoidal rule, of course. Then

$$
\left\|\frac{\partial}{\partial z} \frac{\delta c}{c}\right\|_{L^{2}}^{2}=\delta x^{T} M \delta x .
$$


Note that we are measuring $\frac{\partial}{\partial z} \frac{\delta c}{c}$ rather than $\frac{\delta c}{c}$ itself. Since $\delta c(0)=0$, a bound on the former implies a bound on the latter, but the choice we've made here weights the more oscillatory velocity perturbations more highly, and thus tends to rotate the gradient in the direction of smoother $\delta c$ 's, with favorable computational consequences.

Now regarding the model as the pair $[x, r]$ (rather than $[c, r]$ ), the model norm is taken to be

$$
\|[\delta x, \delta r]\|_{M}^{2}=\mu_{c} \delta x^{T} M \delta x+\mu_{r}\|\delta r\|_{1}^{2}
$$

Adjustment of the weights $\mu_{c}, \mu_{r}$ allows the gradient to be rotated in the $x$ - or $r$-directions; this scaling-preconditioning turns out to be important in achieving rapid convergence.

\subsection{Gradients, Hessians}

First examine the incoherency component of $\tilde{J}$, as before. To write the result in a revealing way, recall that $\delta C$ is linear in $[\delta x, \delta r]$, and write $\delta C \cdot[\delta x, \delta r]$ for the value. Then the limit on the l.h.s. of (23) can be carried out to give

$$
\begin{gathered}
2 \sigma^{2}\langle\delta C \cdot[\delta x, \delta r], C[x, r]\rangle_{L^{2}}= \\
\quad\left\langle[\delta x, \delta r], 2 \sigma^{2} \delta C^{*} \cdot C[x, r]\right\rangle_{M}
\end{gathered}
$$

where the adjoint operator $\delta C^{*}$ is defined by the condition

$$
\langle\delta C[\delta x, \delta r], F\rangle_{L^{2}}=\left\langle[\delta x, \delta r], \delta C^{*} \cdot F\right\rangle_{M}
$$

which is to hold for arbitrary model perturbations $[\delta x, \delta r]$ and $(t, p)$-sections $F$.

Comparison of (23) and (24) reveals that

$$
\begin{aligned}
\operatorname{grad}\left(\sigma^{2}\left|\|C \mid\|^{2}\right)\right. & =[\dot{x}, \dot{r}] \\
& =2 \sigma^{2} \delta C^{*} \cdot C .
\end{aligned}
$$

Likewise, the Gauss-Newton approximate Hessian operator (Dennis and Schnabel (1983), §10.2) is given by

$$
\operatorname{Hess}\left(\sigma^{2}\|C \mid\|^{2}\right) \cdot[\delta x, \delta r]=2 \sigma^{2} \delta C^{*} \cdot \delta C \cdot[\delta x, \delta r] .
$$

Similar formulas hold for the term $\left\|S-S_{\text {data }}\right\| \|^{2}$.

The calculations (26) are the principal parts of the quasi-Newton methods to be introduced below. Thus efficient and accurate calculation of the adjoints $\delta C^{*}, \delta S^{*}$ are essential to successful optimization. 


\subsection{Adjoints}

The definition (25) of $\delta C^{*}$ must be taken absolutely seriously. That is, even though $\delta C$ is given (in principle) by an enormous matrix, $\delta C^{*}$ is not simply the operator defined by the matrix transpose of $\delta C . \delta C^{*}$ is related to the matrix transpose, however, and this relation provides a convenient avenue for computing $\delta C^{*}$.

The scalar products involved in (25) may be written symbolically in the form

$$
\langle X, Y\rangle_{G}=X^{T} G Y
$$

where $X$ and $Y$ are parameter vectors and $G$ is the Gram matrix of $\langle,\rangle_{G}$. Thus $G$ is a positive-definite symmetric matrix of size equal to the dimension of the parameter space.

In (25), two essentially different inner products are involved, on two different parameter spaces (models $[\delta x, \delta r]$, sections $F$ ), as well as a linear transformation $(\delta C)$ mapping one parameter space into the other. Accordingly, consider two inner products of the form given above, with Gram matrices $G_{\nu}, \nu=1,2$, and a linear transformation $A$, given by a matrix of appropriate dimensions, mapping one parameter space into the other. The adjoint of $A$ is defined by the abstraction of (25):

$$
\left\langle A X_{1}, X_{2}\right\rangle_{G_{1}}=\left\langle X_{1}, A^{*} X_{2}\right\rangle_{G_{2}}
$$

for arbitrary $X_{\nu}$ in the $\nu^{\text {th }}$ parameter space, $\nu=1,2$. Written in matrix form,

$$
\left(A X_{1}\right)^{T} G_{1} X_{2}=X_{1}^{T} G_{2} A^{*} X_{2}
$$

from which it is clear that, as matrices,

$$
A^{*}=G_{2}^{-1} A^{T} G_{1} .
$$

To see what (27) implies for (25), write $\delta C$ in components:

$$
\delta C=\left[\delta_{x} C, \delta_{r} C\right] .
$$

This is the correct matrix representation if the model perturbation is written as a column vector:

$$
\delta C \cdot\left[\begin{array}{l}
\delta x \\
\delta r
\end{array}\right]=\delta_{x} C \cdot \delta x+\delta_{r} C \cdot \delta r .
$$

Thus

$$
\delta C^{*}=\left(\begin{array}{c}
\delta_{x} C^{*} \\
\delta_{r} C^{*}
\end{array}\right)
$$

where

$$
\left\langle\delta_{x} C \cdot \delta x, F\right\rangle_{L^{2}}=\delta x^{T} M\left(\delta_{x} C^{*} \cdot F\right)
$$


and

$$
\left\langle\delta_{r} C \cdot \delta r, F\right\rangle_{L^{2}}=\left\langle\delta r, \delta_{r} C^{*} \cdot F\right\rangle_{1} .
$$

Identify a section $F$ with a vector in any convenient fashion, e.g. by listing the traces (columns) sequentially. Then the $L^{2}$-inner product, discretized by the trapezoidal rule, is realized by the scaling matrix $S$ (which scales the edge entries by $1 / 2$, the corner entries by $1 / 4$, and everything else by 1 ):

$$
\left\langle F_{1}, F_{2}\right\rangle_{L^{2}}=F_{1}^{T} S F_{2}
$$

for any sections $F_{1}, F_{2}$.

Thus (27) applied to (28a) gives

$$
\delta_{x} C^{*}=M^{-1} \delta_{x} C^{T} S .
$$

For (28b), it is necessary to write the "Sobolev" inner product $\langle,\rangle_{1}$ in the canonical form given above; its Gram matrix turns out to be exactly the matrix of the discrete Neumann problem for the usual five-point discretization of the Laplace operator, which we shall denote by $N$. Thus

$$
\delta_{r} C^{*}=N^{-1} \delta_{r} C^{T} S .
$$

In principle, this completes the calculation of the adjoints, hence of the gradient and Hessian. In practice, inspection of $(26 \mathrm{a}, \mathrm{b})$ shows that we need only routines which apply $\delta C^{*}$ to a section, not the entire matrix of $\delta C^{*}$. This extremely important observation saves much computational effort and storage. In fact, application of the trapezoidal scaling operation represented by $S$ is trivial, and the transpose operations $\delta_{x} C^{T}, \delta_{r} C^{T}$ are relatively easy to work out, as the same sort of recurrence rules that form the "forward" calculations of $\delta_{x} C, \delta_{\tau} C$. Thus we represent $(29 \mathrm{a}, \mathrm{b})$ in the alternate forms: for an arbitrary section $F$,

$$
\begin{aligned}
\delta_{c} C^{*} \cdot F & =\dot{x} \\
M \dot{x} & =\delta_{x} C^{T} S F \\
\delta_{r} C^{*} \cdot F & =\dot{r} \\
N \dot{r} & =\delta_{r} C^{T} S F
\end{aligned}
$$

We have just described how to compute the right-hand sides of the second equations in each of these pairs. To solve the linear system with the spline mass matrix $M$, we used a standard linear equation solver (LINPACK: SPOFA, SPOSL), as the spline space is small-dimensional-i.e. the background model has few degrees of freedom, $\leq 20$ in all of our experiments. To solve the discrete Neumann problem, which is quite large ( $n t=300, n p=40$ in some experiments), we took advantage of explicit knowledge of the discrete Neumann eigenfunctions (tensorproduct cosines) to design an FFT-based discrete Neumann solver, which solves the second member of $(30 \mathrm{~b})$ very efficiently. 
This step would have been more difficult if the factor $p$ had been included in the integral defining $\langle,\rangle_{1}$, as it should be. Then $N$ would include a $p$ difference approximation to the Bessel operator of order zero, with Neumann condition at $p=p_{\max }$ (a residue of the cylindrical geometry implicit in the proper definition of the Radon transform). Thus fast Bessel transform software would be required.

\subsection{Interface with Optimizer}

Since the optimizer described below accepts standardized $n$-vector arguments, it was necessary to "bundle" the computations just described into procedures with standard calling sequences. We used a pack/unpack routine, which collapses the spline/section pair $[\delta x, \delta r]$ into a vector of length $n s p l+(n t+1) * n p$, and vice versa.

\subsection{Choice of Optimizer}

The tests reported below were made using a so-called truncated Newton code. This code is based on the model trust region principle (Dennis and Schnabel (1983), section 6.4) and on the extensions to it introduced by Steihaug in his Yale thesis (Steihaug (1981)). Essentially, the Gauss-Newton linear step

$$
\text { Hess } \tilde{J} \cdot[\delta x, \delta r]=-\operatorname{grad} \tilde{J}
$$

is solved by a conjugate residual iteration (Golub and Van Loan (1983), Ch. 10), which is terminated when the step estimate exits a ball about the current solution estimate, the radius of which is determined by a simple and robust updating strategy. This expedient avoids expensive conjugate residual steps taken outside the region in which the linearized model can be "trusted", hence the name. A more lengthy description of the code can be found in Santosa and Symes [1986], Chapter 9 , where the same codes were used in solving the nonlinear output least-squares problem, using finite difference synthetic seismograms instead of the convolutional model.

An important amendment of the trust region idea is natural in this problem. All models in the iteration are supposed to have a fixed rectangle $\left[0, t_{\max }\right] \times$ $\left[0, p_{\max }\right]$ as a precritical set. This may cease to be true during an update step, if the velocity is increased by too much at some depth. The computation of the gradient simply flags this occurence, and the algorithm attempts a smaller step in the same direction. Thus the trust region, for problems like the present one, may involve in a natural way constraints on the validity of the model itself. 


\subsection{Numerical Experiments}

We performed a number of numerical experiments using the velocity profile $c$ (upper curve) and perturbation $\Delta c$ (lower curve) exhibited in Figure 6 to generate the $p$-tau convolutional model data of Figure 8 , by convolving with a Ricker wavelet with center frequency $20 \mathrm{~Hz}$. A target background velocity with a lower velocity zone was chosen because the structure of such a zone is impossible to determine from refraction arrival times, and intrinsically more difficult for least-squares methods - see Santosa and Symes [1986]. The velocity, hence the slowness, were normalized against the surface velocity, by changing the measure of depth to normal-incidence time for a constant background velocity equal to the surface velocity. This step also normalizes the slowness to the range $0 \leq p \leq 1$. A happy side-effect of this normalization was to reduce substantially the numerical imprecision resulting from mis-scaling inherent in the use of physical units.

The algorithm explained in the preceding subsections was used to extract estimates of $c$ and $r$ from the data of Figure 8. Parameters common to all experiments were

$$
\sigma=1, \quad, \mu_{c}=10^{-4}, \quad \mu_{r}=1 .
$$

We found the small value of $\mu_{c}$ necessary to rotate the gradient of $\tilde{J}$ toward the "c-"direction.

In all cases, we observed the same pattern. We began with the simple estimate $c_{\text {initial }}=$ const. $(=1), r_{\text {initial }} \equiv 0$. The first Newton step did not change the estimate of $c$, since the incoherence of $r \equiv 0$ vanishes for any velocity model. Otherwise put, since there are initially no reflectors, there is initially no moveout information in the reflectivity with which to update the velocity model. The first iteration is thus devoted entirely to minimizing

$$
\left\|f * \frac{\partial r}{\partial t}-S_{\text {data }}\right\| \|^{2}
$$

which amounts to deconvolving the data in a least-squares sense to find an initial (nontrivial) estimate for $r$. Unless otherwise noted, each Newton step (including the first) is approximated by five conjugate-residual iterations.

In the second and subsequent iterations, the velocity model is improved, and data-noise-generated incoherency in the reflectivity is reduced.

Figure 9 shows the velocity-estimate results of five and ten Newton steps steps from both the constant velocity model (curve 0 ) and five Newton steps from the velocity model identified as the curve 1 . The results are labeled as curves 2,3 and 4 respectively. In all cases we used 8 spline nodes (thus the velocity is determined by 5 parameters). This result is evidence for the independence of initial estimate of the final estimates. In particular, the results of 
ten iterations from a constant initial model, and five iterations from the "incorrect trend" initial model (curve 1) are virtually identical. The error is quite stable and is due to the fact that the target is not a member of the space of velocities defined by the eight-node spline basis - i.e. we get the "closest" eight-node velocity estimate.

These estimates are reasonably accurate, especially considering the computational work required. Apparently the incoherence resulting from the erroneous basement velocity was insufficient to cause further corrections, or possibly the calculation of the incoherence is substantially inaccurate there - see comments below. Most of the reduction in $\tilde{J}$ (about an order of magnitude) came in the first iteration, in which the data is deconvolved. Yet another order of magnitude is gained in the remaining iterations, in which the incoherence is reduced.

Two major points have emerged from the experimental work conducted thus far. The first concerns the number of spline nodes: the outcome is in some ways quite sensitive to this number. For example, the experiment of Figure 9 was repeated with 16 nodes instead. Five, ten, and thirty iterations of the Gauss-Newton process produced the curves labeled 1, 2, and 3 in Figure 10. These look quite "wild," and certainly the mean-square error is much greater than is the case with those in Figure 9. Recall however that the principal role of the background model is to supply travel-times. A quite different picture emerges when the travel-times are plotted against the "true" travel time curve (from the velocity profile of Figure 6). In Figure 11 are displayed (normalincidence) travel-time curves from the "exact" velocity, curve 1 from Figure 9, and curves 1 and 2 from Figure 10. In fact, the latter two curves are closer to the "true" travel-time than is the former, despite their correspondence with velocity estimates having larger $L^{2}$-error. This relation emerges more clearly when the (normal incidence) travel-time errors are plotted: see Figure 12.

Apparently, the result of increasing the number of degrees of freedom in the model is to allow a closer fit to to the travel-time, at least at points corresponding to major reflectors, but at the cost of an oscillatory error which may be large in the mean-square sense. This is easy to understand: the errors oscillate on a length scale too short to affect the travel-times between major reflectors, hence correspond to small eigenvalues of the incoherence Hessian. While the effect on travel-times of this sort of error is a priori small, it does produce irritating ambiguities in the velocity estimate, and (more important) has a negative impact on the convergence of the iterative scheme. Several approaches to the removal of this ambiguity suggest themselves. Trial-and-error determination of the optimal spacing for spline nodes, as has been done here, is clearly unsatisfactory. Systematically increasing the number of nodes until a good fit is obtained requires some notion of an "acceptable" level of fit, and such information may itself only be obtainable by trial-and-error. A more satisfactory approach might be adaptive estimation of small Hessian eigenvalues, through 
the close relation of conjugate-residual iteration with the Lanczos algorithm (Golub and Van Loan [1983], Ch. 10), and penalization of the corresponding eigenvector components. For the application of such "iterative deflation" to linear systems see Chan [1986] (also Meza and Symes [1987]). Since the number of small eigenvalues associated with velocity perturbations is small, and since their characterization is somewhat independent of the current velocity estimate, this deflation strategy should work rather well in combination with Gauss-Newton iteration. Finally, since the culprit is oscillatory error, penalization of a velocity derivative may regularize this problem satisfactorily. Computational trials are in progress; results will be reported elsewhere.

A second point concerned the density of p-samples. Inspections of Figure 8 clearly show that for large $p$ and $t$, the moveout difference in neighboring traces may be a substantial fraction of a wavelength. As we have based our difference approximations to the incoherency on centered difference approximations to the coordinate derivatives $\frac{\partial}{\partial t}, \frac{\partial}{\partial p}$, the possibility exists of severe undersampling in $p$. In fact when we increased $\Delta p$ to .02 (from .01 as in Figure 8), the computation analogous to that for Figure 9 gave completely erroneous results for the deeper part of the velocity profile, apparently because the part of the incoherency due to deeper reflectors was grossly underestimated. We suspect that residual inaccuracy in the deeper parts of the curves in Figure 9 is due to a milder version of the same effect.

Besides finer sampling, methods to overcome errors in incoherency due to undersampling include higher-order difference formulas and difference formulas better adapted to the moveout. Indeed, low-order differences along even a crude approximation to the correct moveout curve should produce better results at coarser sampling than the coordinate derivatives used in our present code. These ideas are also under investigation.

It might be objected that, while the output includes an estimate of the travel-time reflectivity section $r(t, p)$, no estimate of the corresponding velocity perturbation $\Delta c(z)$ is provided. The final reflectivity is not necessarily entirely coherent, and so does not correspond to any $\Delta c(z)$, strictly speaking. Nonetheless, an estimate may be produced by stacking $r(t, p)$ on the basis of equation (5), i.e.

$$
\Delta c(z) \sim \frac{c^{3}(z)}{p_{\max }} \int_{0}^{p_{\max }} d p v^{-2}(z, p) r(\tau(z, p), p) .
$$

This output might truly be regarded as the final image produced by an iterative before-stack migration, specialized to constant-density acoustics.

We have implemented this post-inversion stack of the final reflectivity estimate, and display the results for the reflectivity corresponding to curve 3 in Figure 9. When stacked with constant-velocity moveout, the estimate of $\Delta c(z)$ is completely erroneous in phase and wrong by a factor of perhaps 3 in amplitude (Figure 13). When stacked with the final velocity estimate from Figure 9 
(curve 3), the estimated $\Delta c(z)$ has essentially correct phases and amplitudes for the major events (Figure 14), as compared to the $\Delta c(z)$ actually used to generate the data. Of course, none of the subwavelength-scale variation in the true $\Delta c(z)$ could appear in the reflectivity $r(t, p)$ or in the stacked estimate, so a more interesting comparison is the $p$-tau section generated using the velocity from Figure 9 and the stacked $\Delta c(z)$ from Figure 14. This is displayed in Figure 15, and the difference of Figure 15 and Figure 8, plotted on the same scale, in Figure 16.

\subsection{Summary}

We have described a preliminary implementation of the coherency optimization method, exhibited the results achievable with this rather crude code, and suggested some issues worthy of further examination. Given the rather large discretization errors in our present implementation, which play the role (at least) of data noise, the stability of the final estimates and the rate of convergence both appear quite satisfactory: to a limited extent, we appear to have satisfied the criteria (a)-(c) stated in Section 2.

Extensive noise studies, tuning, modifications along the lines suggested above, and direct comparison with output least-squares optimization will be reported in a future publication.

\section{Coherency Optimization for Laterally Heterogeneous Models}

The essential ingredients of the approach to velocity inversion sketched in Section 2 were:

(i) parameterization of the reflectivities as time "sections" (i.e. traces), so that the seismogram is a regular function of the reflectivities, one reflectivity (trace) per plane-wave "shot" (synthetic source);

(ii) referral of each time-section reflectivity to depth, and assessment of the dependence of the resulting suite of depth sections on the "shot" parameter (i.e. slowness).

In this section, we maintain the fiction that the seismogram is adequately approximated by the multi-dimensional version of the convolutional model, i.e., the linearization about a smooth background velocity, and moreover apply highfrequency asymptotics freely. A natural interpretation of (i) and (ii) emerges in this context. 
First recall why the parameterization by two-way time resulted in regularity of the seismogram as a function of both the background velocity and the reflectivity. In effect, each reflector was associated with the time of arrival, at the surface, of its reflection. If the background velocity is changed, then depth-parameterized reflectors remain fixed while their reflection times change, whereas time-parameterized reflectors remain fixed while their depths change. In the former case, high-frequency arrivals are time-shifted, while in the latter case they are not. The time-shift is a time derivative in the infinitesimal limit, and its appearance marks the loss of regularity of the depth-parameterized reflectivity-to-travel-time map. For time-parameterized reflectivities, no such time shift occurs as a result of background velocity change, and regularity is maintained.

Reflectors and reflection arrivals are both (near)-singularities, i.e. locii of high-frequency energy. In the high-frequency limit, therefore, we must ask: how can we parameterize reflectivity so that a singularity in the reparameterized reflectivity corresponds, under conversion to ordinary spatial coordinates and mapping to the seismogram, to a singularity in the same location? In severaldimensional problems, singularities may have orientations, and these must be preserved as well.

This question is answered by a theorem of Rakesh (Symes (1985), Rakesh (1988)), together with a construction presented for this problem by Beylkin (1985). To fix ideas, suppose we consider the linearized acoustic problem for a point source, which models a shot-gather:

$$
\frac{1}{c^{2}} \frac{\partial^{2} u}{\partial t^{2}}-\nabla^{2} u=\frac{2 r}{c^{2}} \frac{\partial^{2} u_{0}}{\partial t^{2}}
$$

where $r=\Delta c / c$ is the "reflectivity", $c$ is the background velocity, $u$ is the scattered field, and $u_{0}$ is the reference field satisfying

$$
\frac{1}{c^{2}} \frac{\partial^{2} u_{0}}{\partial t^{2}}-\nabla^{2} u_{0}=f(t) \delta\left(\underline{x}-\underline{x}_{s}\right)
$$

$\underline{x}$ being the source location. Rakesh showed that for the impulsive case $(f(t)=$ $\vec{\delta}(t))$ a singularity in $r$ at the subsurface location $y$, across a surface element with normal $\underline{\eta}$, corresponds to a singularity in the reflected field at receiver location $\underline{x}_{r}$, time $t_{r}$, only if there exist

- an incident ray $\gamma_{i}$ associated with the reference field, emanating from the source-point $\underline{x}$, at $t=0$;

- a reflected ray $\gamma_{r}$, passing over the receiver point $\underline{x}_{r}$ at time $t_{r}$

so that $\gamma_{i}$ and $\gamma_{r}$ meet at the reflector point $\underline{y}$ at some intermediate time, making equal-angles with the reflector normal $\underline{\eta}$. For non-impulsive but broad- 
band time signatures $f(t)$, this rule governs the arrival of (primary-reflected) high-frequency energy.

This is exactly the usual picture of the reflection process, of course. Rakesh's theorem, which justifies this picture in terms of the wave equation, holds in complete generality, so long as the background velocity $c$ is smooth. It tells us exactly which singularity in the time section must be mapped to a given singularity in the depth section if the seismogram is to return such singularities to their original position and orientation. Beylkin's construction, on the other hand, applies only when no caustics exist in the incident field, i.e. each depth point $y$ is joined to the source point $\underline{x}_{s}$ by a unique incident ray. Then any mapping having the singularity-moving properties just described must differ only by a (possibly frequency-dependent) amplitude modulation (technically, a pseudodifferential operator) from the Kirchhoff migration formula

$$
\begin{aligned}
r\left(\underline{y}, \underline{x}_{s}\right) & =K \tilde{r}\left(\underline{y}, \underline{x}_{s}\right) \\
& =\int d \underline{x}_{r} w\left(\underline{x}_{s}, \underline{y}, \underline{x}_{r}\right) \tilde{r}\left(\underline{x}_{r}, \tau\left(\underline{x}_{s}, \underline{y}, \underline{x}_{r}\right), \underline{x}_{s}\right) .
\end{aligned}
$$

Here $\tau\left(\underline{x}_{s}, \underline{y}, \underline{x}_{r}\right)$ is the two-way reflection phase, i.e. the time from $\underline{x}_{s}$ to $\underline{y}$ to $\underline{x}_{r}$, and $w\left(\underline{x}_{r}, \underline{x}_{\theta}, \underline{y}\right)$ is a slowly-varying amplitude modulation.

Thus: the reflectivity time-sections $\tilde{r}\left(\underline{x}_{r}, t, \underline{x}_{g}\right)$ will be converted to reflectivity depth sections $r\left(\underline{y}, \underline{x}_{0}\right)$ via a Kirchhoff migration formula like (31). This implicitly defines the seismogram as a function of $\tilde{r}\left(\underline{x}_{r}, t, \underline{x}_{s}\right)$, and guarantees that it is regular as a function of $c, \tilde{r}$. Note the apparent similarity of (31) to the travel-time change-of-variables, appropriate in the layered case (formula (A.1), for instance).

The second ingredient (ii) in the coherency optimization approach is evidently the condition that the depth-parameterized reflectivities $r\left(\underline{y}, \underline{x}_{s}\right)$ are actually independent of $\underline{x}_{\text {s }}$ ("Every shot sees the same earth"). Regarding the source locations as filling up a continuum, this amounts to the condition $\nabla_{\underline{x}} r\left(\underline{y}, \underline{x}_{s}\right)=0$. The composition rules for oscillatory integrals (the local calculus of Fourier Integral operators-e.g. Duistermaat (1975), Ch. 2) give the result:

$$
\nabla_{\underline{x}} r=\nabla_{\underline{x}} K \tilde{r}=K\left(\nabla_{\underline{x}}+P\right) \tilde{r}
$$

up to an error rapidly decaying in frequency content, where $P$ is a pseudodifferential operator in $\underline{x}_{r}$ and $t$, i.e. an oscillatory integral of the form

$$
\operatorname{Pr}\left(\underline{x}_{r}, t\right)=\iint d \underline{x} d s d \underline{k} d \omega e^{i\left[\underline{k} \cdot\left(\underline{x}_{r}-\underline{x}\right)+\omega(t-s)\right]} p\left(\underline{x}_{r}, t, \underline{x}, s, \underline{k}, \omega\right) \tilde{r}(\underline{x}, s) .
$$

The amplitude $p$ depends on the ray geometry, i.e. on the background velocity $c$, but the phase $\left.\underline{k}_{\left(x_{r}\right.}-\underline{x}\right)+\omega(t-s)$ does not. Thus $\left(\nabla_{\underline{x}_{\mathrm{o}}}+P\right) \tilde{r}$ is regular as a function of $c$, i.e. perturbing $c$ does not result in the appearance of higher derivatives of $\tilde{r}$, just as was the case for the formula (A.1) for $C[c, r]$. 
Now let $L$ be any operator from depth-parameterized reflectivities to sections which has the reverse effect to $K$ on singularities: for instance $L$ might be taken as the linearized seismogram operator itself. Then another application of the same reasoning shows that

$$
C[c, \tilde{r}]:=L \nabla_{\underline{x}}, K \tilde{r}
$$

is regular as a function of $c, \tilde{r}$ for the same reason. We take this formula as our definition of the incoherency for the laterally heterogeneous acoustic problem. Note that $L$ must be computed to form the seismogram, and $K$ is the Kirchhoff migration operator (or an equivalent). Thus $C[c, \tilde{r}]$ involves only well-understood computations. Also, the astute reader will note the immediate resemblance to the definition of $C[c, r]$ given in Section 2 .

We conclude that both main ingredients of coherency optimization, as explained in Section 2 for layered acoustics, generalize in an acceptable way to a simple laterally heterogeneous model. Many details remain to be settled, some of which will doubtless be crucial to computational efficiency. Also, the analysis of Section 3 remains to be generalized. A technical complication is that, while the operators $L$ and $K$ exist in general, the composition rules leading to the regularity of the incoherency $C[c, \tilde{r}]$ must be modified when caustics are present. Nonetheless, we have established that the coherency approach is not conceptually restricted to layered problems.

To end this section, note again that $\tilde{r} \rightarrow K \tilde{r}$ serves the role of the travel-time transformation. The first recognition of the special role of travel-time in regularizing 1-d problems was probably the work of Gray (1980), as noted above. Some time later, Gray and Hagin (1984) attempted generalized travel-time coordinates for laterally heterogeneous point source problems, with limited success; travel-time (ray-straightening) coordinates per se do not exist in general for several-dimensional problems. Nonetheless, the operator $K$ accomplishes the principal effect of the 1-d travel-time coordinate, i.e. to make reflector location independent of background velocity in both the (reparameterized) reflectivity and in the seismogram simultaneously. Of course, $K$ is a more complicated operator than a change of coordinates, except for 1-d (plane-wave, layered model) problems.

\section{Discussion and Conclusion}

\subsection{The scope of the coherency approach}

From a theoretical point of view, and perhaps from a practical one as well, the chief defect in the results of Sections 2 and 3 on the layered acoustic problem is the absolute lack of any provision for multiple reflections. This defect is cured 
in the companion paper Symes [1988], in which the fully nonlinear bandlimited layered velocity inversion problem is treated with full mathematical rigor. We reach a qualitatively identical conclusion about the appropriate version of coherency optimization: that is, it gives a (regularized) solution of the inverse problem stably dependent on near-consistent data, provided that sufficiently many reflectors are present, i.e. that the target profile is sufficiently rough. We give a precise sense for "rough", and a relation emerges between stability, aperture, bandlimits, and roughness (reflector density) very similar to that explained in Section 3.

Both convolutional model and fully nonlinear versions of other layered inverse problems should succumb to the same approach. We mention specifically nonconstant-density acoustics, the "marine" elastic model (with sources and receivers in an overlying fluid layer), and either of these with the source timedependence and directivity also regarded as unknowns. Some idea of the novel features of these problems in convolutional approximation may be gleaned from Sacks and Symes [1987] and Bube, Lailly, Sacks, Santosa, and Symes [1987]. No technical obstacles appear to lie in the way of an analogous treatment of these problems.

Note that the density, regarded as independent of velocities, will not be recovered with trend, in any of these problems, as density trend perturbations do not affect ray geometry. This gross density ambiguity is well-known (Tarantola 1986) and has been observed in output-least-squares results (Canadas and Kolb, 1986).

The program outlined in Section 5 appears feasible. On the other hand, while an analogous coherency optimization principle can be formulated for fully nonlinear laterally heterogeneous models, its analysis will require fundamental advances in the understanding of wave propagation in rough media.

\subsection{Conclusion}

We have presented a novel approach to the reflection seismic inverse problem, which has its roots in utterly commonplace concepts in seismic data processing. We have formulated this coherency optimization principle precisely for the convolutional approximation to the layered constant-density acoustic model, and suggested both analytically and numerically that its solution yields accurate and stable estimates of both velocity trends and reflectivities, to the extent that these are determined by the precritical plane-wave data set used. Our analysis indicated that coherency optimization should require markedly less computational effort than output-least-squares inversion. Finally, we formulated an analogous principle for laterally heterogeneous velocity inversion, a problem for which output-least-squares inversion is so inefficient as to be infeasible. It remains to be seen whether coherency optimization yields a computationally 
tractable approach to such several-dimensional problems. 


\section{Appendix. Computation of a derivative}

This appendix details the calculation of the derivative of the incoherency

$$
\begin{aligned}
C[c, r] & =\left[\frac{\partial}{\partial p}\left(v^{-2} r \circ \tau\right)\right] \circ \zeta \\
& =-2 p r+\left[v^{-2} \frac{\partial}{\partial p}(r \circ \tau)\right] \circ \zeta \\
& =-2 p r+v^{-2} \circ \zeta\left(\frac{\partial r}{\partial p}-p \frac{\partial r}{\partial t} \int_{0}^{\zeta} v\right)
\end{aligned}
$$

in which have been used the identities

$$
\begin{gathered}
\frac{\partial}{\partial p} v^{-2}=-2 p, \quad \frac{\partial v}{\partial p}=p v^{3} \\
\frac{\partial \tau}{\partial p}=-p \int_{0}^{z} v .
\end{gathered}
$$

Clearly

$$
\delta C=\delta_{c} C+\delta_{r} C
$$

with

$$
\delta_{r} C=C[c, \delta r] .
$$

On the other hand, an easy calculation shows that

$$
\delta \zeta=v \circ \zeta \int_{0}^{\zeta} v \frac{\delta c}{c^{3}}
$$

So from the third line in (A.1)

$$
\begin{aligned}
\delta_{c} C= & \delta\left(v^{-2} \circ \zeta\right)\left[\frac{\partial}{\partial p}(r \circ \tau)\right] \circ \zeta \\
& -p v^{-2} \circ \zeta \frac{\partial r}{\partial t} \delta \int_{0}^{\zeta} v \\
= & -2\left[\frac{\delta c}{c^{3}}+\frac{1}{c^{3}} \frac{\partial c}{\partial z} c \int_{0}^{z} v \frac{\delta c}{c^{3}}\right] \circ \zeta\left[\frac{\partial}{\partial p}(r \circ \tau)\right] \circ \zeta \\
& -p\left[\int_{0}^{z} v^{-2} \Lambda \delta c+v^{-2} \int_{0}^{z} \Lambda \delta c\right] \circ \zeta \frac{\partial r}{\partial t}
\end{aligned}
$$

An alternate form of (A.2) follows from the identity

$$
\begin{aligned}
\delta \int_{0}^{\zeta} v & =\delta\left(\int_{0}^{t}(v \circ \zeta)^{2}\right) \\
& =2 \int_{0}^{t}(v \circ \zeta) \delta(v \circ \zeta)=2 \int_{0}^{\zeta} \delta(v \circ \zeta) \circ \tau .
\end{aligned}
$$

So, using the notation

$$
\begin{aligned}
Q \delta c & =\delta(v \circ \zeta) \circ \tau \\
& =\Lambda^{3}\left(\delta c+\frac{\partial c}{\partial z} v \int_{0}^{z} v \frac{\delta c}{c^{3}}\right)
\end{aligned}
$$


the first line in (A.2) may be re-written

$$
\begin{aligned}
\delta_{c} C= & -2\left[v^{-3} Q \delta c \frac{\partial}{\partial p}(r \circ \tau)\right] \circ \zeta \\
& -2 p v^{-2} \circ \zeta \frac{\partial r}{\partial t} \int_{0}^{\zeta} Q \delta c .
\end{aligned}
$$

For section 3 we require an evaluation of $\delta_{c} C$ when $r$ is coherent, i.e. (5) is satisfied. Then for suitable $\Delta c$,

$$
r=\left(\Lambda^{2} \frac{\Delta c}{c}\right) \circ \zeta
$$

Moreover, from (A.1),

$$
\frac{\partial}{\partial p}(r \circ \tau) \circ \zeta=2 p\left(v^{2} \circ \zeta\right) r
$$

so $\left(\mathrm{A} .2^{\prime}\right)$ becomes

$$
\begin{aligned}
\delta_{c} C=-2 p & \left\{2\left[Q \delta c \cdot v^{-1}\right] \circ \zeta \cdot r+\left[v^{-2} \int Q \delta c\right] \circ \zeta \frac{\partial r}{\partial t}\right\} \\
=-2 p & \left\{2(Q \delta c) v c^{-3} \Delta c+\left(\int Q \delta c\right) v^{-1} \frac{\partial}{\partial z}\left(\Lambda^{2} \frac{\Delta c}{c}\right) \circ\right\} \\
=-2 p & \left\{2(Q \delta c) \Lambda c^{-2} \Delta c\right. \\
& \left.+\left(\int Q \delta c\right)\left(2 \Lambda^{3} c^{\prime} p^{2}\left(\frac{\Delta c}{c}\right)+c^{-1} \Lambda \frac{\partial}{\partial z}\left(\frac{\Delta c}{c}\right)\right)\right\} \circ \zeta \\
=-2 p & \left\{2\left[(Q \delta c) \Lambda c^{-1}+\left(\int Q \delta c\right) p^{2} \Lambda^{3} c^{\prime}\right]\left(\frac{\Delta c}{c}\right)\right. \\
& \left.+\left[\left(\int Q \delta c\right) c^{-1} \Lambda\right] \frac{\partial}{\partial z}\left(\frac{\Delta c}{c}\right)\right\} \circ \zeta .
\end{aligned}
$$

For our computations, we used the formulas (A.1) and (A.2') with

$$
\left(\frac{\partial}{\partial p}(r \circ \tau)\right) \circ \zeta
$$

computed as in the last line of (A.1). 


\section{References.}

BAMBERGER, A., G. CHAVENT, C. HEMON, and P. LAILLY [1982]. Inversion of normal incidence seismograms, Geophysics 47 , pp. 757-770.

BEYLKIN, G. [1985]. Imaging of discontinuities in the inverse scattering problem by inversion of a causal generalized Radon transform, J. Math. Phys. 26, pp. 99-108.

BUBE, K., P. LAILLY, P. SACKS, F. SANTOSA, and W. SYMES [1987]. Simultaneous determination of source wavelet and velocity profile using impulsive point-source data from a layered fluid, Geophys. J. Roy. Astr. Soc., to appear.

BUBE, K., D.B. JOVANOVICH, R.T. KANGAN, J.R. RESNICK, R.T.SHUEY, and D.A. SPINDLER [1985]. Well-determined and poorly determined features in seismic reflection tomography: Part II, 55th Annual Meeting, SEG, Washington, DC.

BEYLKIN, G. and R. BURRIDGE [1987]. Linearized inverse scattering problems of acoustics and elasticity, preprint; also Multiparameter inversion for acoustic and elastic media, Expanded Abstract, 57th Annual International Meeting, Society of Exploration Geophysicists, New Orleans, pp. 747-749.

CANADAS, G. and P. KOLB [1986] Least-squares inversion of prestack data: simultaneous identificatio of density and velocity of stratified media, Expanded abstract, 56th Annual International Meeting, Society of Exploration Geophysicists, Houston, pp. 604-607.

CHAN, T. [1985]. Deflated Lanczos procedures for solving nearly singular linear systems, research report YALEU/DCS/RR-403, Department of Computer Science, Yale University.

CLAYTON, R. and R. STOLT [1981]. A Born-WKBJ inversion method for acoustic reflection data, Geophysics, 46, pp. 1559-1567.

COHEN, J.K. and N. BLEISTEIN [1979]. Velocity inversion procedure for acoustic waves, Geophysics 44, pp. 1077-1085.

DENNIS, J. E. JR. and R. B. SCHNABEL [1983]. Numerical Methods for Unconstrained Optimization and Nonlinear Equations, Prentice-Hall, Englewood Cliffs.

DUISTERMAAT, J. [1975]. Fourier Integral Operators, Courant Institute Lecture Notes. 
FOWLER, P. [1986] Migration velocity analysis by optimization: linear theory, Expanded Abstract, 56th Annual International Meeting, Society of Exploration Geophysicists, Houston, pp. 660-662.

GAUThIER, O. , TARANTOLA, A. and VIRIEUX, J. [1986]. Two-dimensional nonlinear inversion of seismic waveforms, Geophysics 51, pp. 1387-1403.

GOLUB, G. and C. VAN LOAN [1983]. Matrix Computations, The Johns Hopkins University Press, Baltimore.

GRAY, S.H. [1980]. A second-order procedure for one-dimensional velocity inversion, SIAM J. Appl. Math. 39, pp. 456-462.

GRAY, S. and F. HAGIN [1984]. Travel-time-like variables and the solution of velocity inverse problems, preprint.

HADJEE, Y. and F. COLLINO [1988]. A geometrical approach to the a-priori study of the 1-d inverse problem, IFP preprint.

IKELLE, L. , J. DIET and A. TARANTOLA [1988]. Linearized inversion of multioffset seismic data in the omega-k domain: depth-dependent reference medium, Geophysics 53, pp. 50-64.

KLEYN, A. H. [1983]. Seismic Reflection Interpretation, Applied Science Publishers, New York.

KOLB P., F. COLLINO, and P. LAILLY [1986]. Prestack inversion of a 1D medium, Proc. IEEE 74, pp.498-506.

LAILLY, P. [1984]. Migration methods: partial but efficient solutions to the seismic inverse problem, in Inverse Problems of Acoustic and Elastic Waves, ed. Santosa et al., SIAM, Philadelphia.

LINES, L. and S. TREITEL [1984]. A review of least-squares inversion and its application to geophysical problems, Geophysical Prospecting 32, pp. 159-186.

LINES, L., J. SCALES and S. TREITEL [1987] (preprint on seismic tomography, to appear in J. Comp. Phys.)

LINES, L., A. SCHULTZ and S. TREITEL [1988]. Coperative inversion of geophysical data, Geophysics 53, pp. 8-20.

MCAULAY [1985]. Prestack inversion with plane-layer point source modeling, Geophysics 50, pp. 77-89.

MEZA, J. and W. SYMES [1987]. Deflated Krylov subspace methods for nearly singular linear systems, Technical Reprot 87-3, Department of Mathematical Sciences, Rice University. 
MORA, P. [1986]. Nonlinear 2-d elastic inversion of multi-offset seismic data, Expanded abstract, 56th Annual International Meeting, Society of Exploration Geophysicists, Houston, pp. 533-537; also Geophysics 52.

MORA, P. [1987]. Nonlinear 2-d elastic inversion of real data, Expanded abstract, 57th Annual International Meeting, Society of Exploration Geophysicists, New Orleans, pp. 430-432.

RAKESH [1988]. A linearized inverse problem for the wave equation, Comm. on P. D. E. 13, pp. 573-601.

SACKS, P. and W. SYMES [1987]. Recovery of the elastic parameters of a layered half-space, Geophys. J. Roy. Astr. Soc. 88, pp. 593-620.

SANTOSA, F. and W. SYMES [1986]. Least-squares principles for layered velocity inversion, to appear in Society of Exploration Geophysicists Monograph Series (1988-89).

SANTOSA, F. and W. SYMES [1987]. Bandlimited Velocity Inversion, Expanded Abstract, 57th Annual International Meeting, Society of Exploration Geophysicists, New Orleans, pp. 437-439.

SPRATT, S. [1987]. Effect of normal moveout errors on amplitude versus offsetderived shear reflectivity, Expanded Abstract, 57 th Annual International Meeting, Society of Exploration Geophysicists, New Orleans, pp.634-637.

STEIHAUG, T. [1981]. Quasi-Newton methods for large-scale nonlinear problems, Ph.D. Thesis, Yale University.

SYMES, W. [1985]. Stability and instability results for inverse problems in several-dimensional wave propagation, Proc. of Seventh Internat. Conf. on Computing Methods in Applied Science and Engineering (INRIA).

SYMES, W. [1988]. Bandlimited Velocity inversion: a model inverse problem from reflection seismology, preprint.

TARANTOLA, A. and B. VALLETTE [1982]. Inverse problems: quest for information, J. Geophys. 50, pp. 159-170.

TARANTOLA, A. [1984]. The seismic reflection inverse problem, in Inverse Problems of Acoustic and Elastic Waves, ed. Santosa et al., SIAM, Philadelphia.

TARANTOLA, A. [1986]. A strategy for nonlinear elastic inversion of seismic reflection data, Geophysics 51, pp.1893-1903. 


\section{FIGURE CAPTIONS}

1. Smooth velocity model (upper curve) and perturbation (lower curve): the latter is non-zero only where the former is constant (this is the situation analysed in section 3). Horizontal scale is one-way time at surface velocity.

2. The coefficient $\lambda$ (display (18)), normalized to remove velocity dependence.

3. The coefficient $\lambda_{1}$ (display (19)), normalized to remove velocity dependence.

4. (a) cubic b-splines: 5 th (solid) and 7th (dashed) of 12 nodes in interval; (b) indefinite integrals of splines in (a).

5. Differences: 6 th -5 th (solid), 7th - 6th (dashed) spline integrals.

6. Smooth velocity model (upper curve) and velocity perturbation (lower curve) used to generate data for coherency optimization experiments.

7. Eigenvalues $\mu_{\max }^{0}, \mu_{\min }^{(1)}$, and $\mu_{\min }$ as in displays (20) and (21).

8. Plane wave section generated from model displayed in Figure 6, using a Ricker wavelet peaked at $20 \mathrm{~Hz}$. 
9. (0) target model, from Figure 6 (solid curve);

(1) a non-constant initial model (short dashes);

(2) result of 5 Gauss-Newton steps starting at constant model (long dashes);

(3) result of 10 Gauss-Newton steps starting at constant model (dash/cross);

(4) result of 5 Gauss-Newton steps starting at model (1) (short/long dashes).

10. Results using too many degrees of freedom in model ( 16 nodes):

(0) target model (solid curve);

(1) Gauss-Newton steps (short dashes);

(2) 10 Gauss-Newton steps (long dashes);

(3) 30 Gauss-Newton steps (short/long dashes)

11. Two-way travel time curves at normal incidence:

(0) target model (solid curve);

(1) curve 2, Figure 9 (short dashes);

(2) curve 1, Figure 10 (long dashes);

(3) curve 2, Figure 10 (short/long dashes).

12. Two-way travel-time errors:

(0) between curves ( 0 ) and (1), Figure 11 (solid line);

(1) between curves ( 0 ) and (2), Figure 11 (short dashes);

(2) between curves (0) and (3), Figure 11 (long dashes). 
13. Stack (solid line) of reflectivity estimate corresponding to Figure 9, curve 2 , at constant velocity, compared with target velocity perturbation (dashed line).

14. Stack (solid line) of reflectivity estimate corresponding to Figure 9, curve 2, at velocity given by Figure 9, curve 2, compared with target velocity perturbation (dashed line).

15. "Synthetic" section produced using velocity from Figure 9, curve 2, with stacked velocity perturbation estimate from Figure 14.

16. Difference of Figures 15 and 8. 


\section{FIGURE 1}

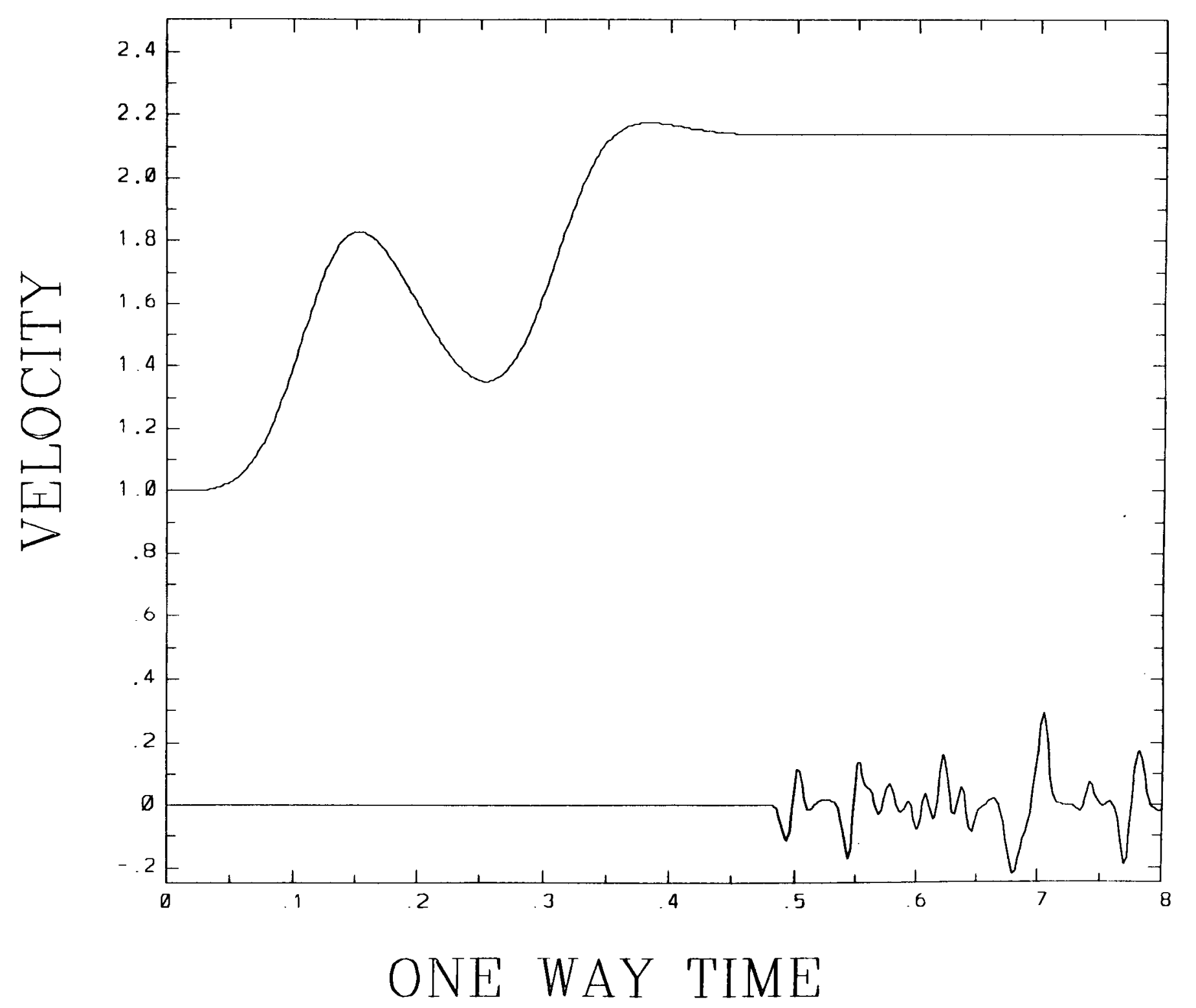




\section{FIGURE 2}

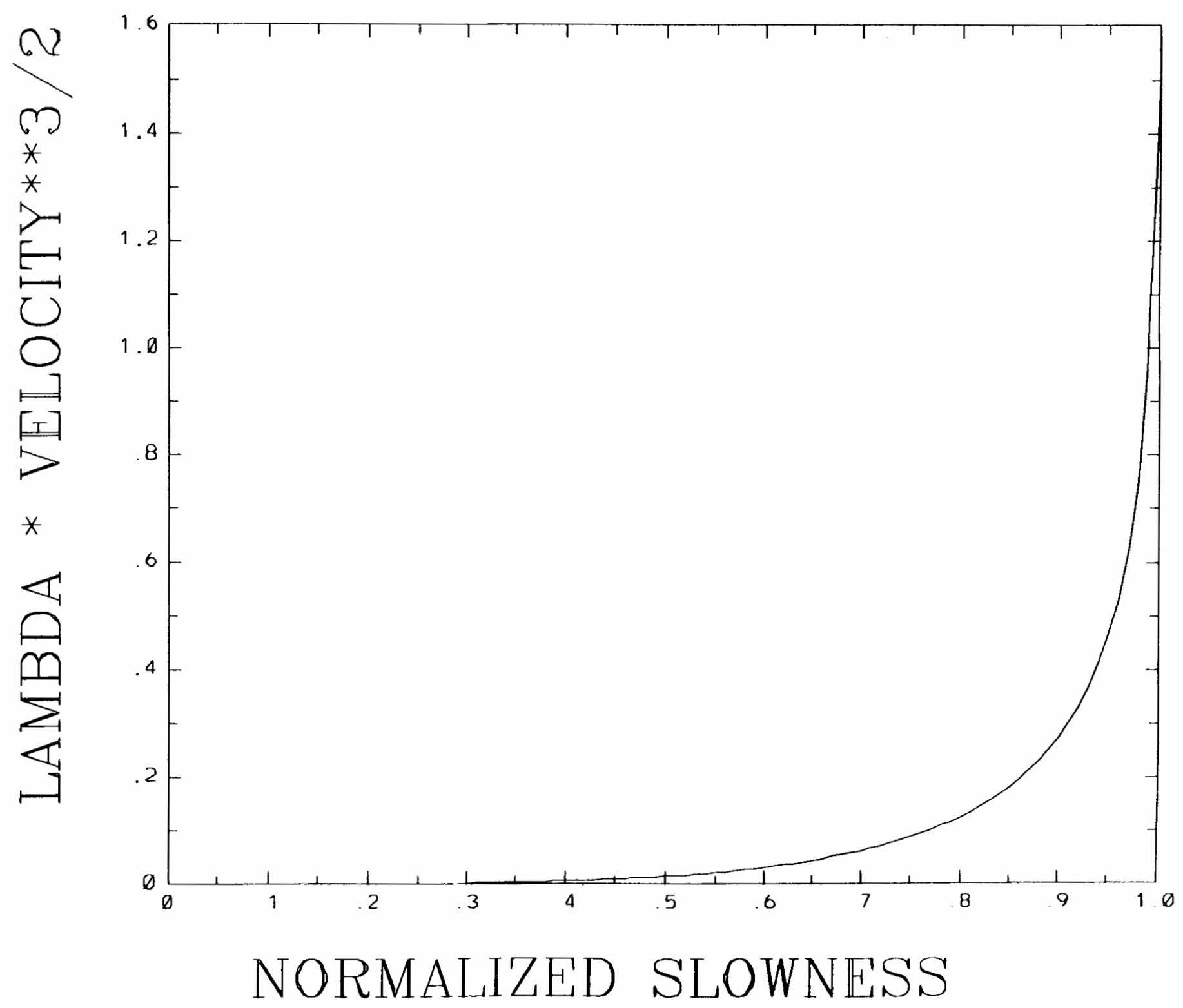


FIGURE 3

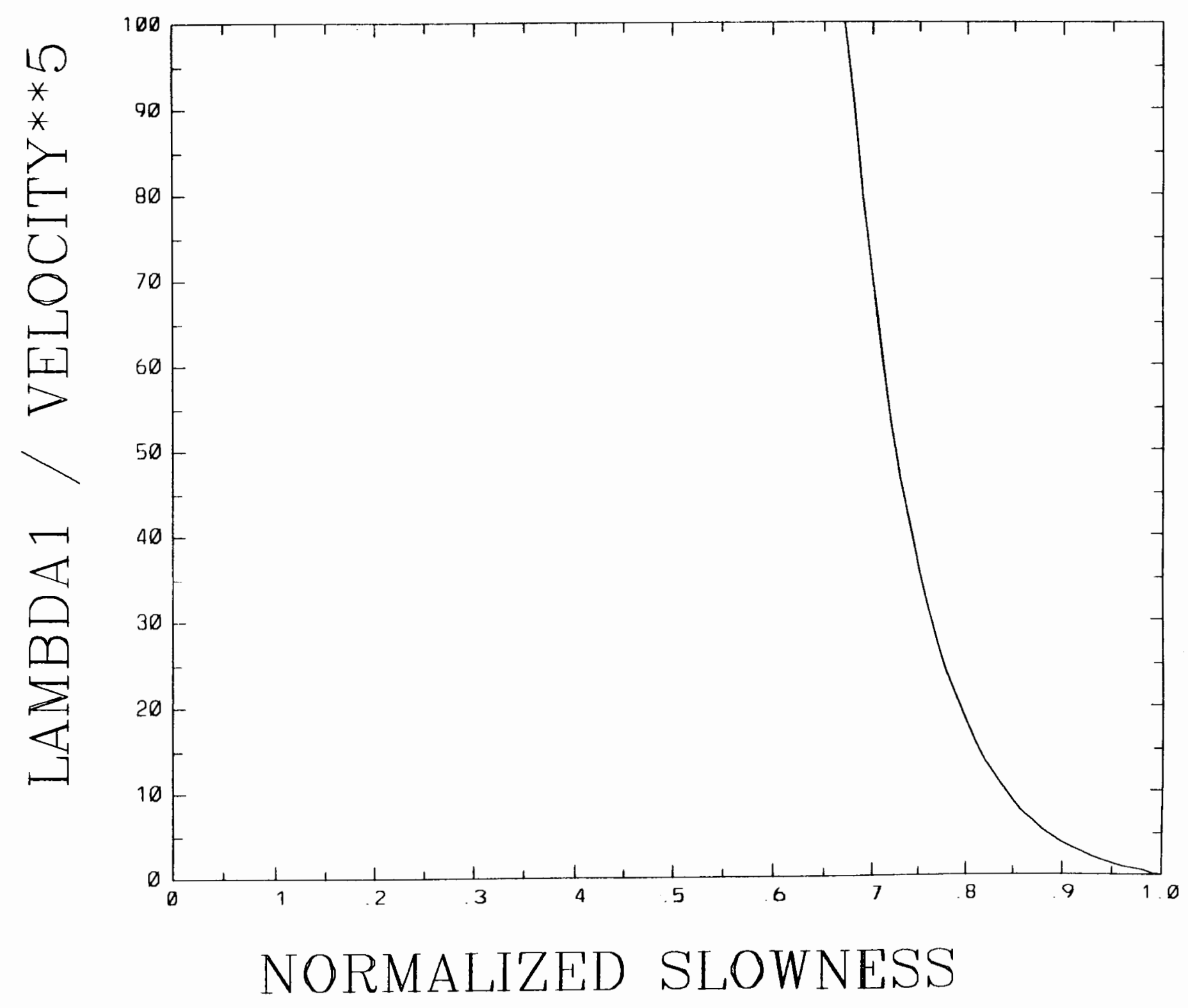




\section{FIGURE 4 A}

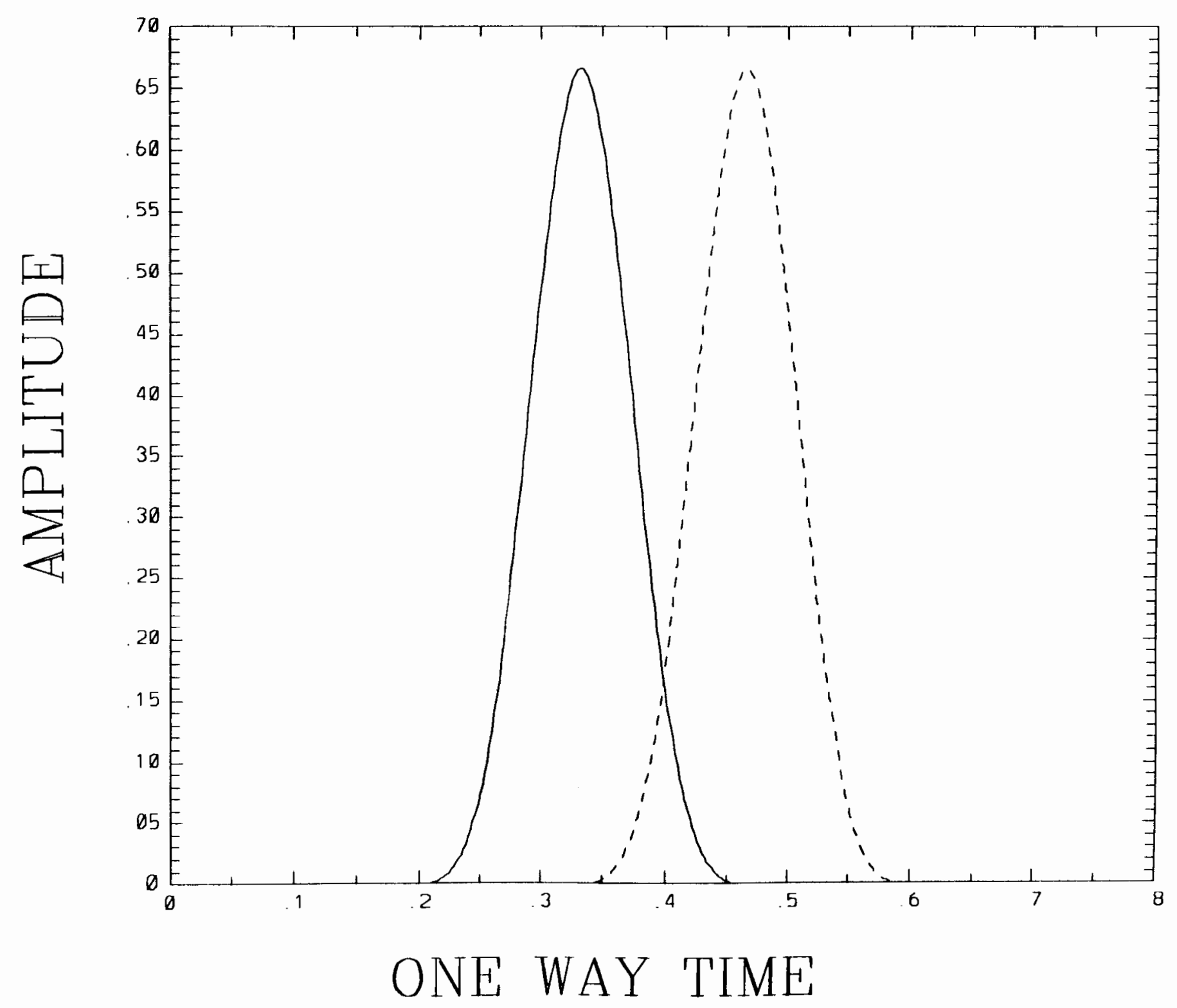




\section{FIGURE 4 B}

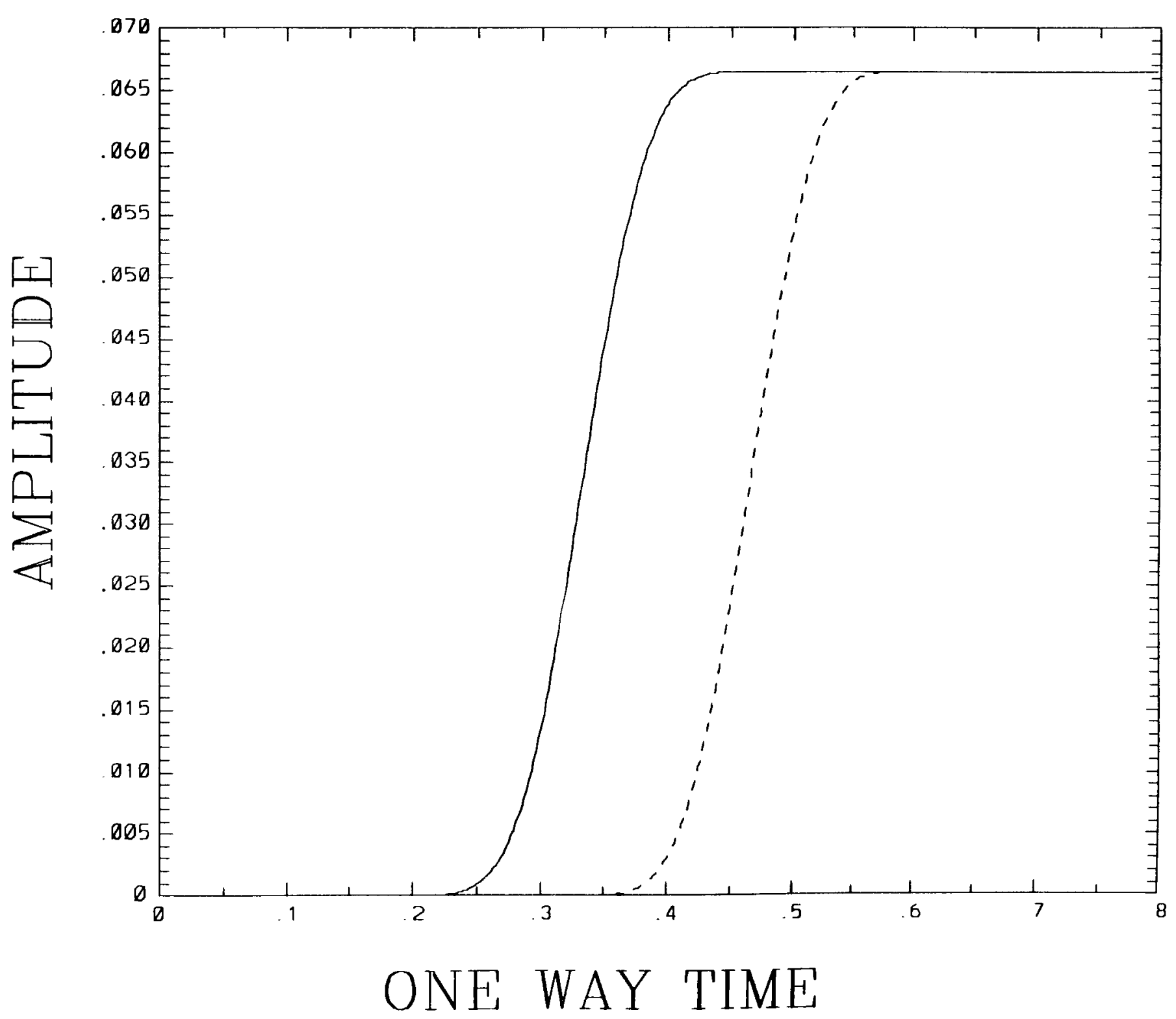




\section{FIGURE 5}

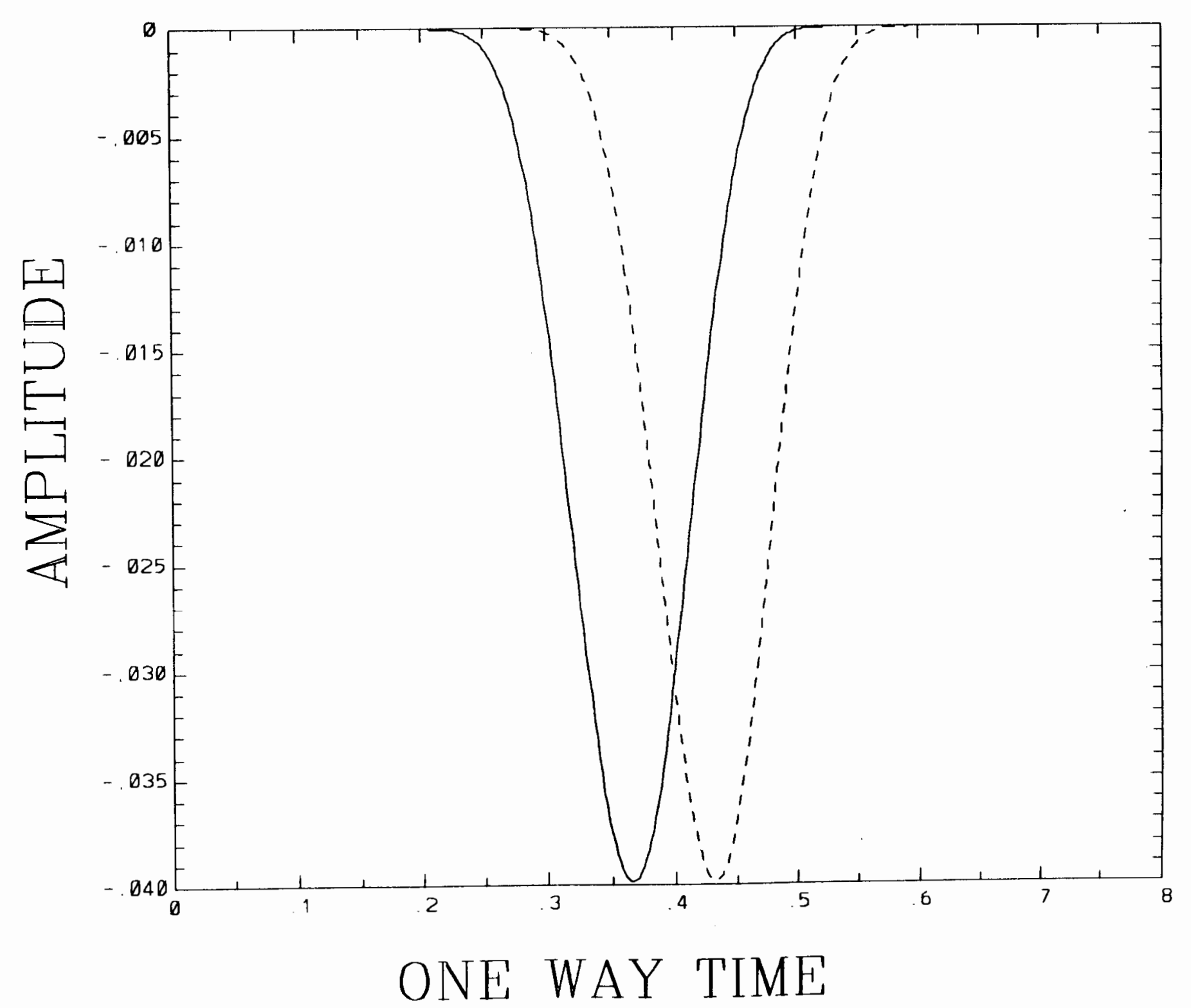




\section{FIGURE 6}

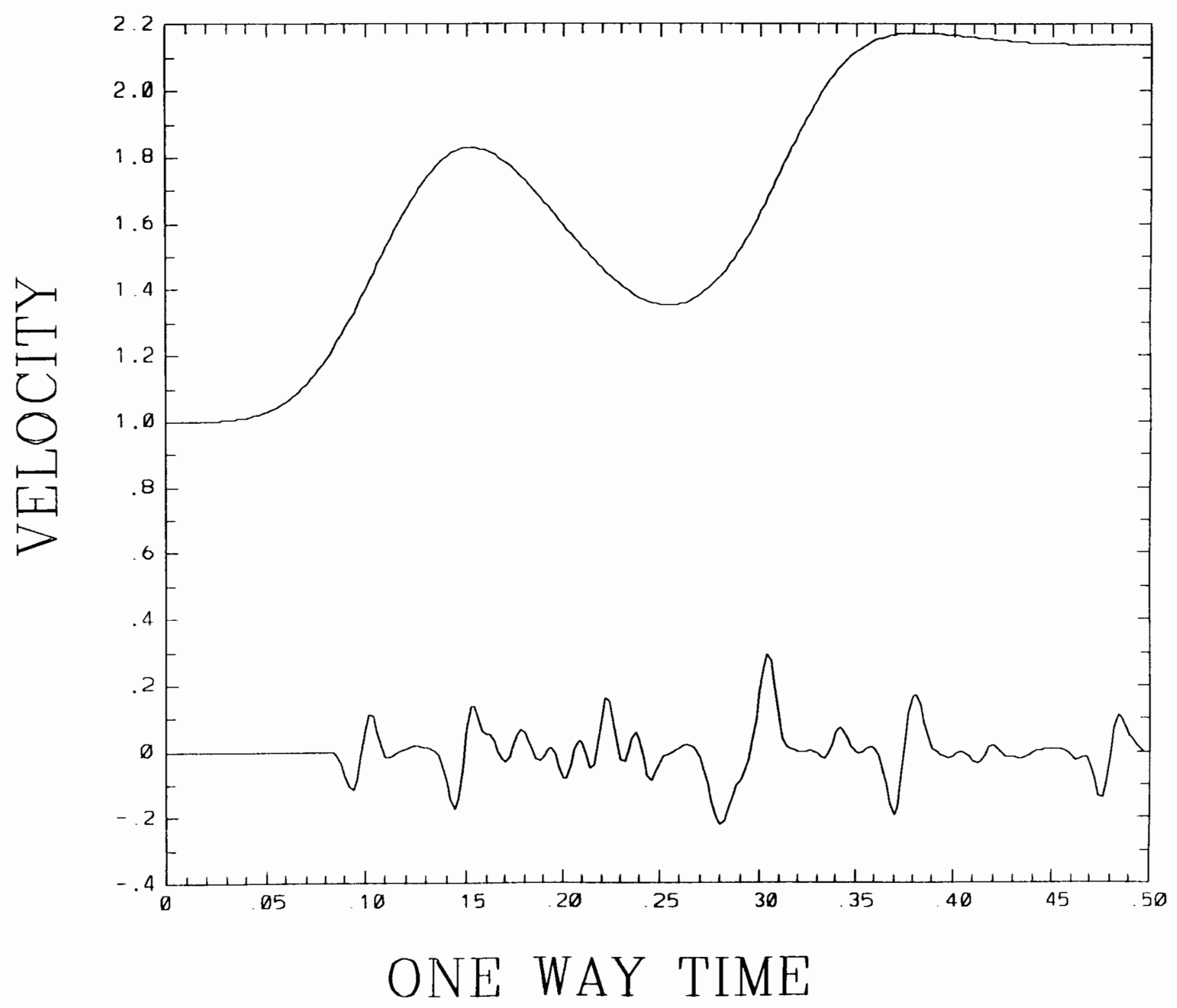


Figure 7

\begin{tabular}{cccc} 
Nodes & $\mu_{\max }^{(0)}$ & $\mu_{\min }^{(1)}$ & $\mu_{\min }$ \\
\hline & & & \\
5 & $.8465 e-02$ & $0.1096 e+01$ & $0.1111 e+01$ \\
6 & $.1305 e-01$ & $0.5551 e+00$ & $0.5741 e+00$ \\
7 & $.1211 e-01$ & $0.3650 e+00$ & $0.3872 e+00$ \\
8 & $.1644 e-01$ & $0.1900 e+00$ & $0.2192 e+00$ \\
9 & $.1798 e-01$ & $0.5334 e-01$ & $0.8287 e-01$ \\
10 & $.1753 e-01$ & $0.3068 e-01$ & $0.4868 e-01$
\end{tabular}




\section{FIGURE 8}

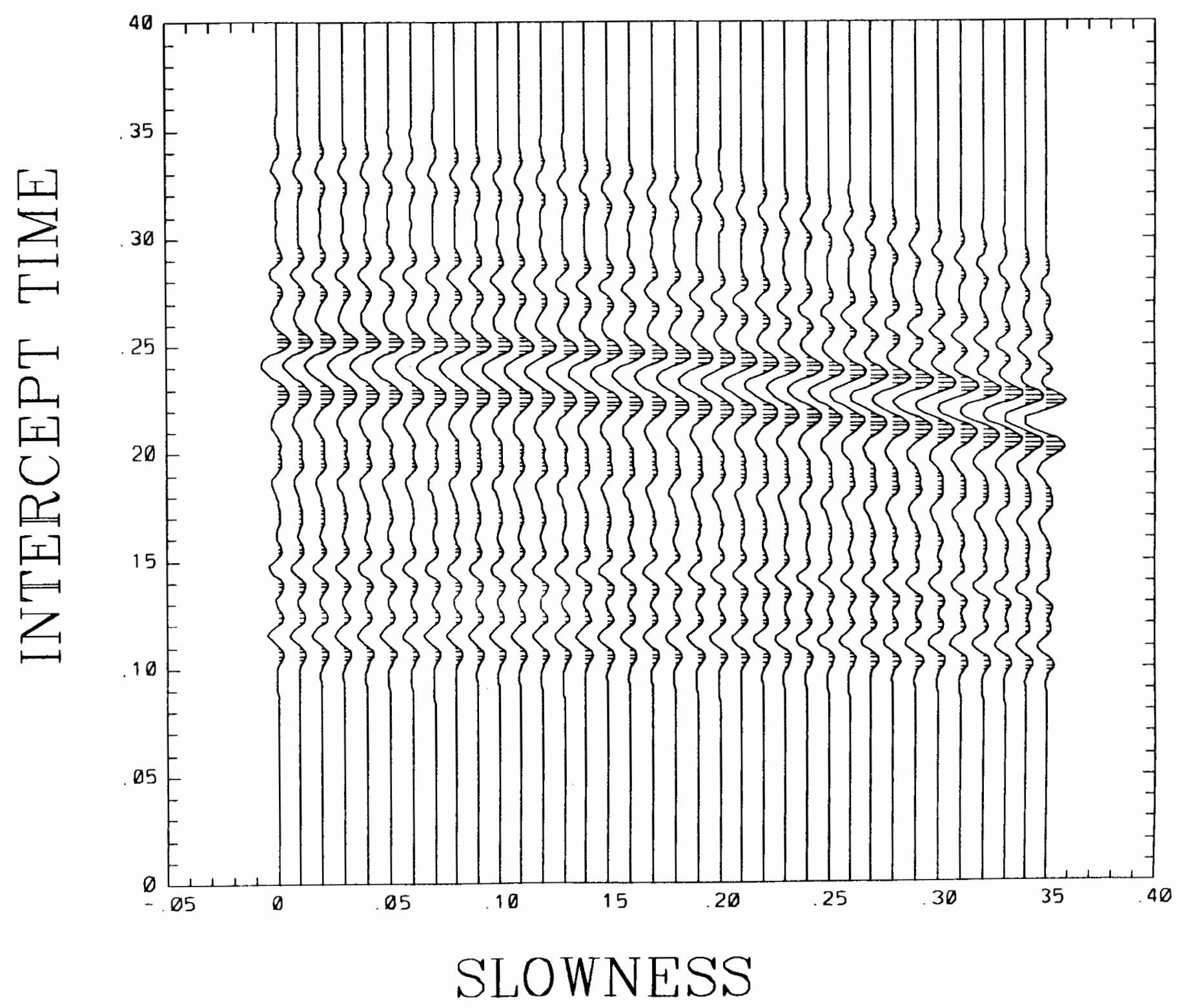




\section{FIGURE 9}

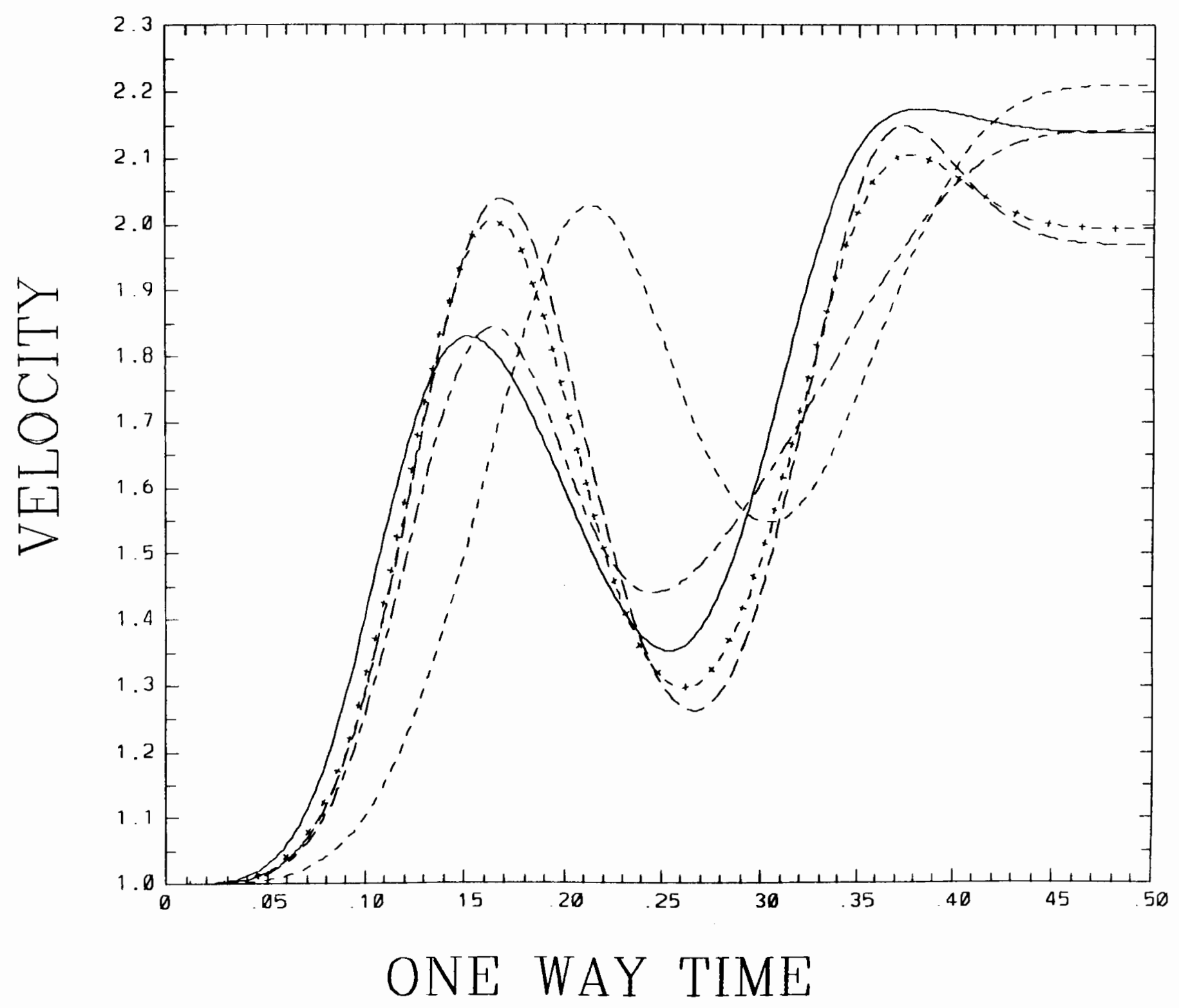




\section{FIGURE 10}

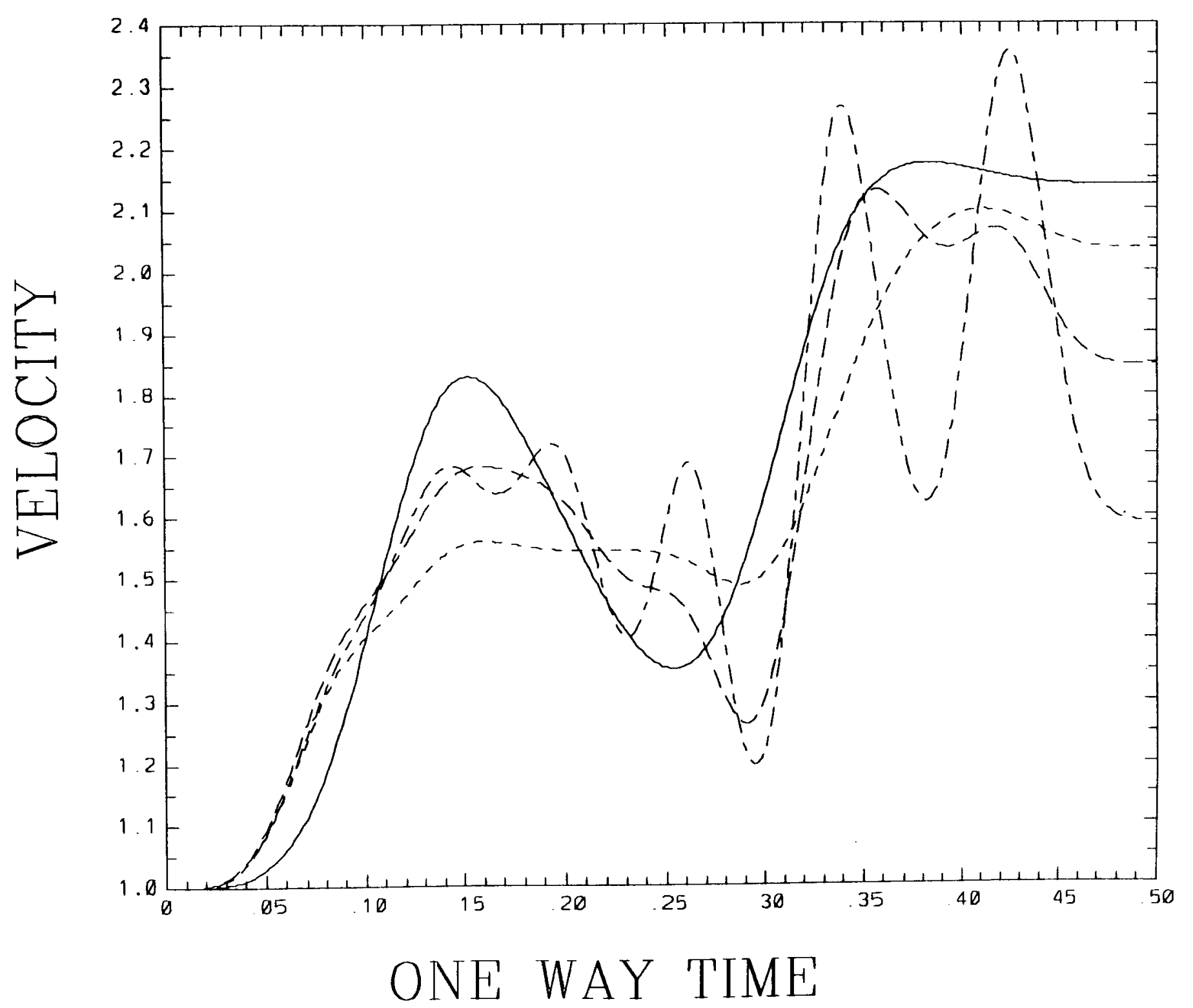




\section{FIGURE 11}

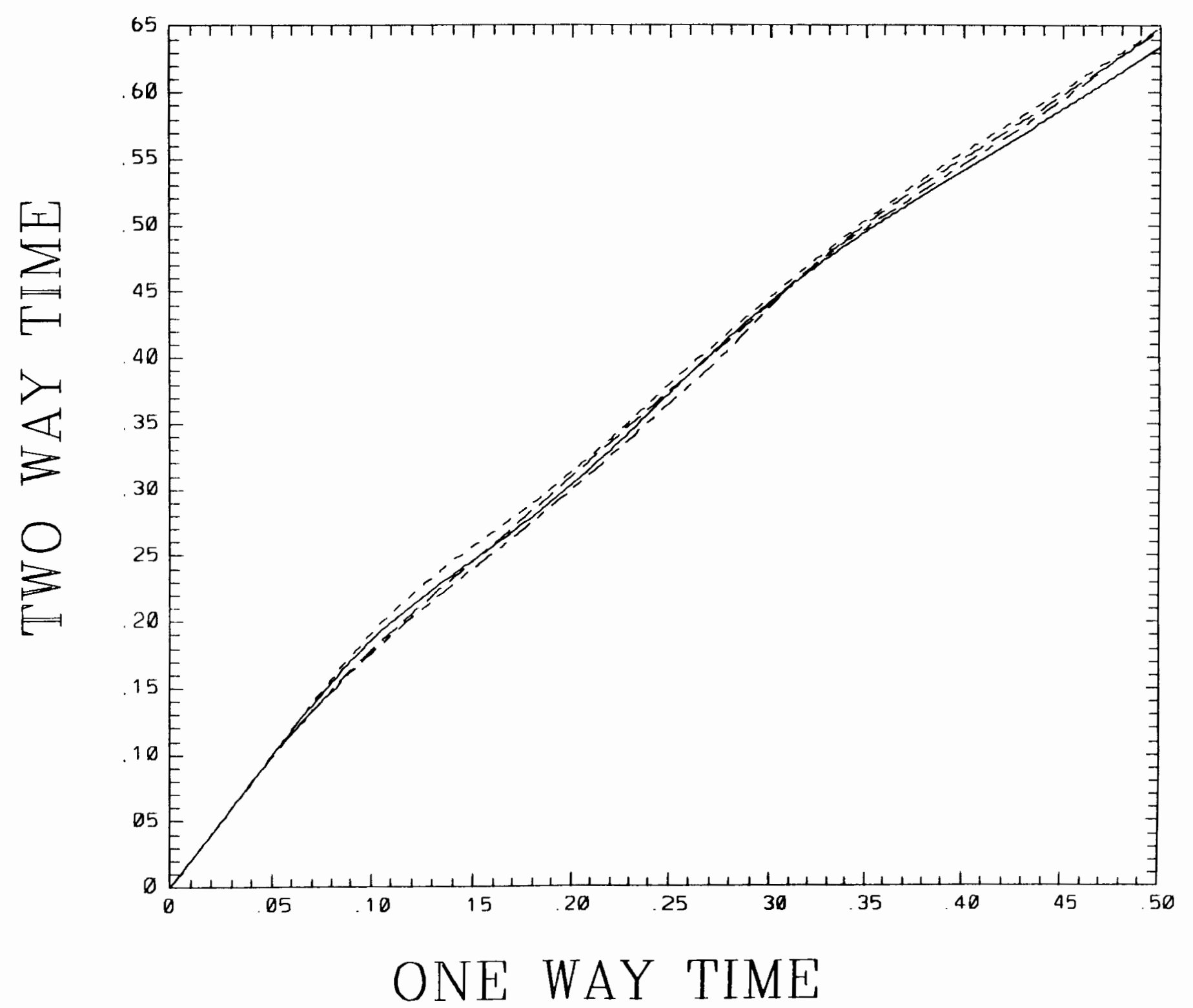




\section{FIGURE 12}

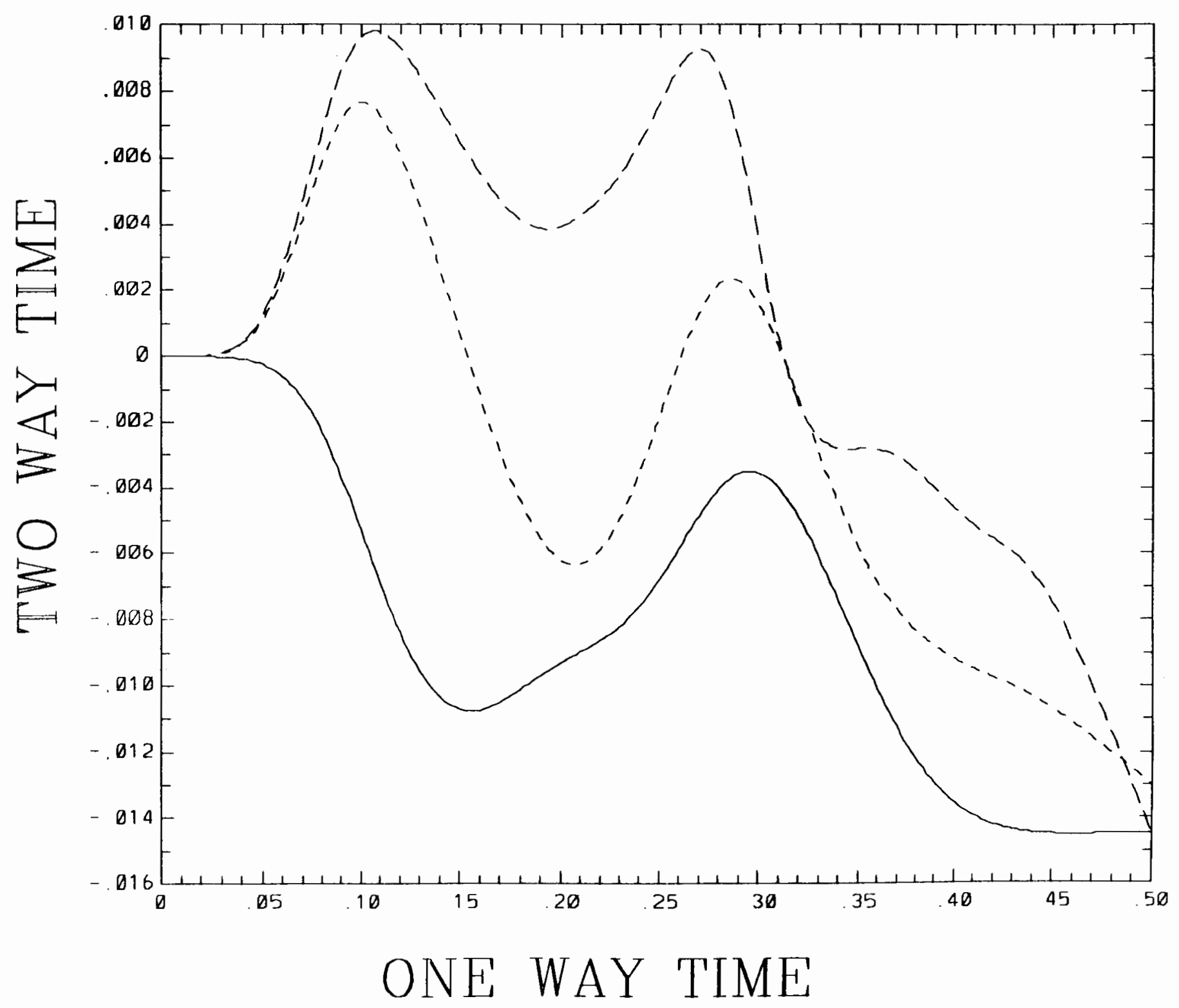




\section{FIGURE 13}

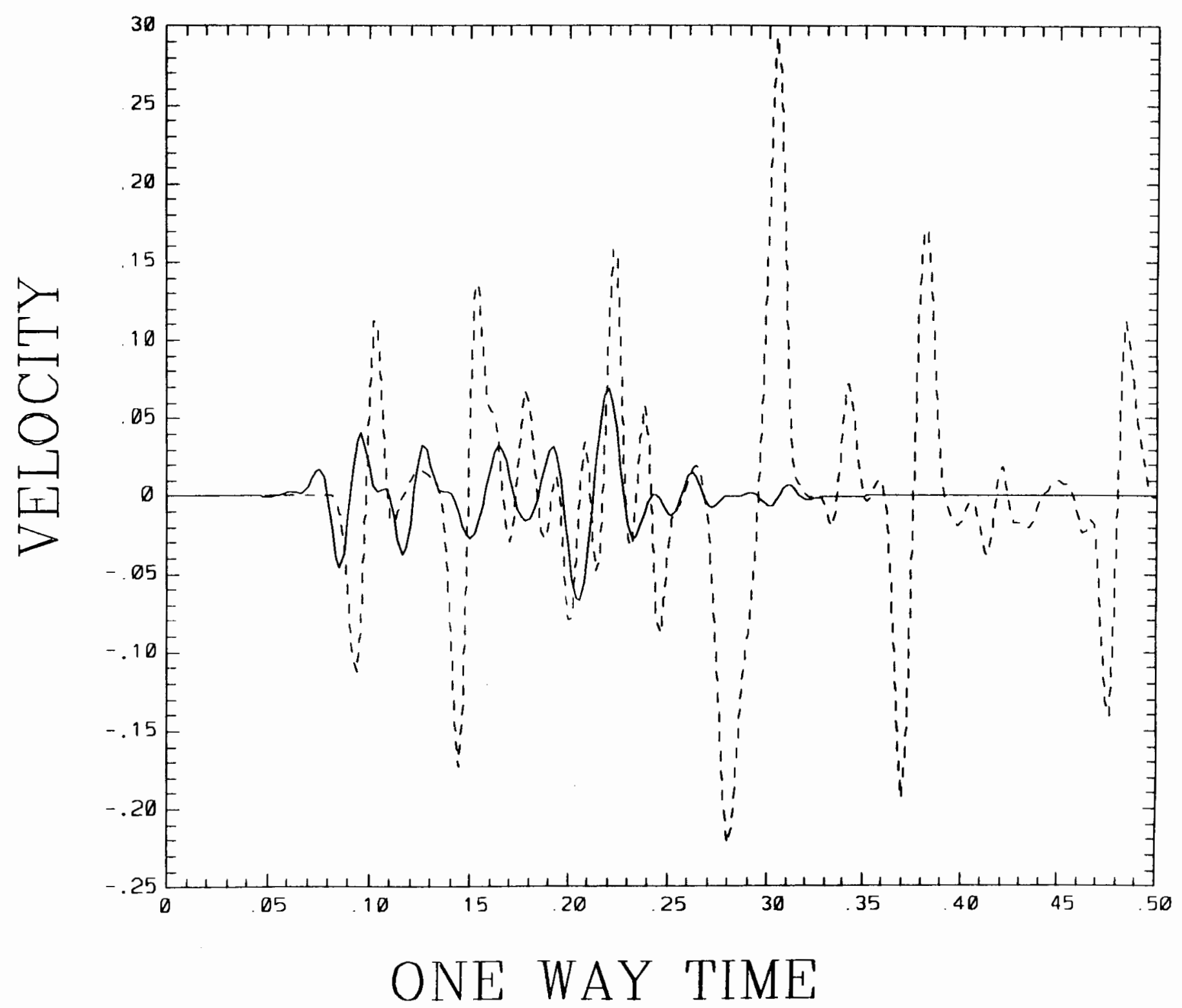




\section{FIGURE 14}

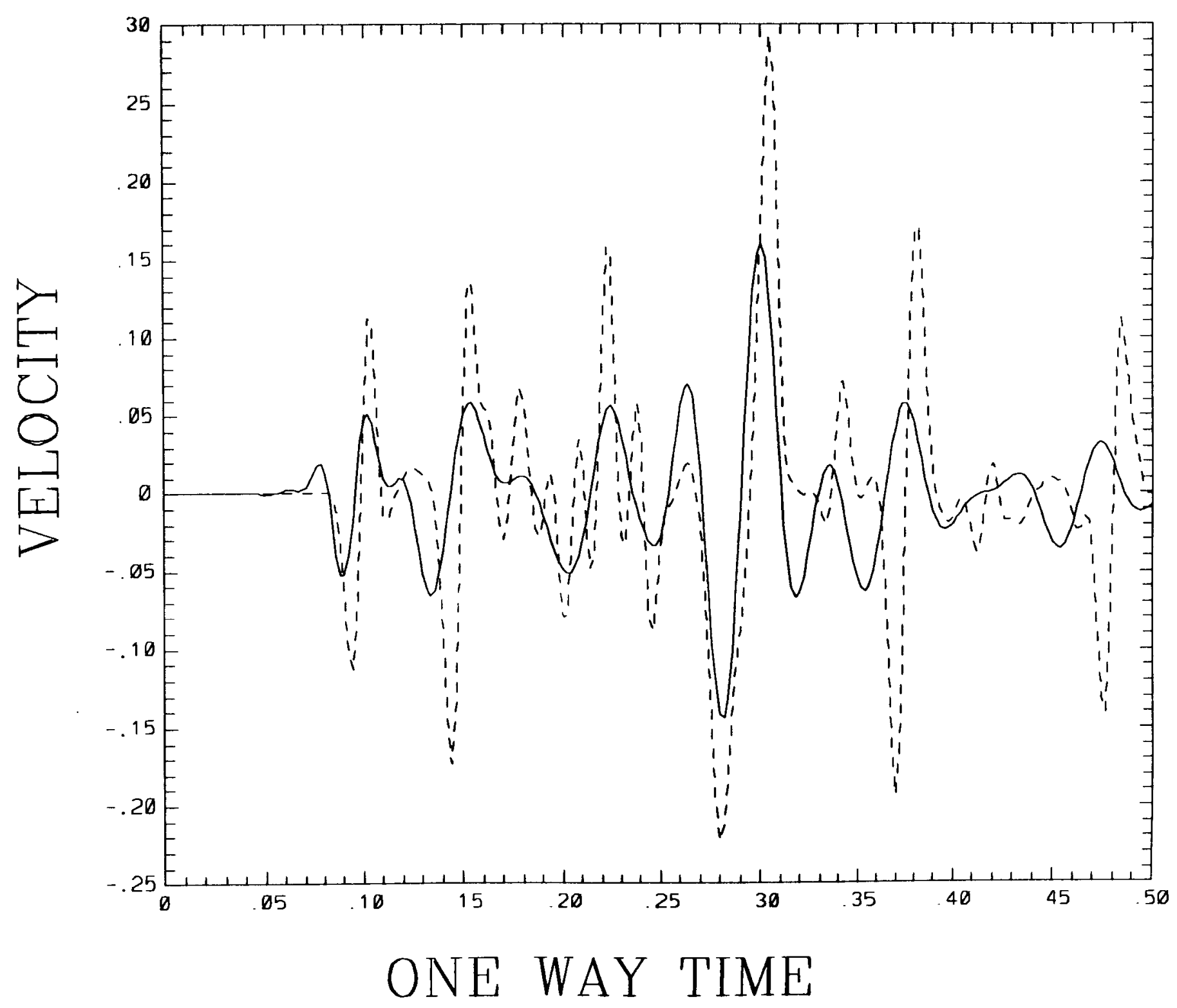




\section{FIGURE 15}

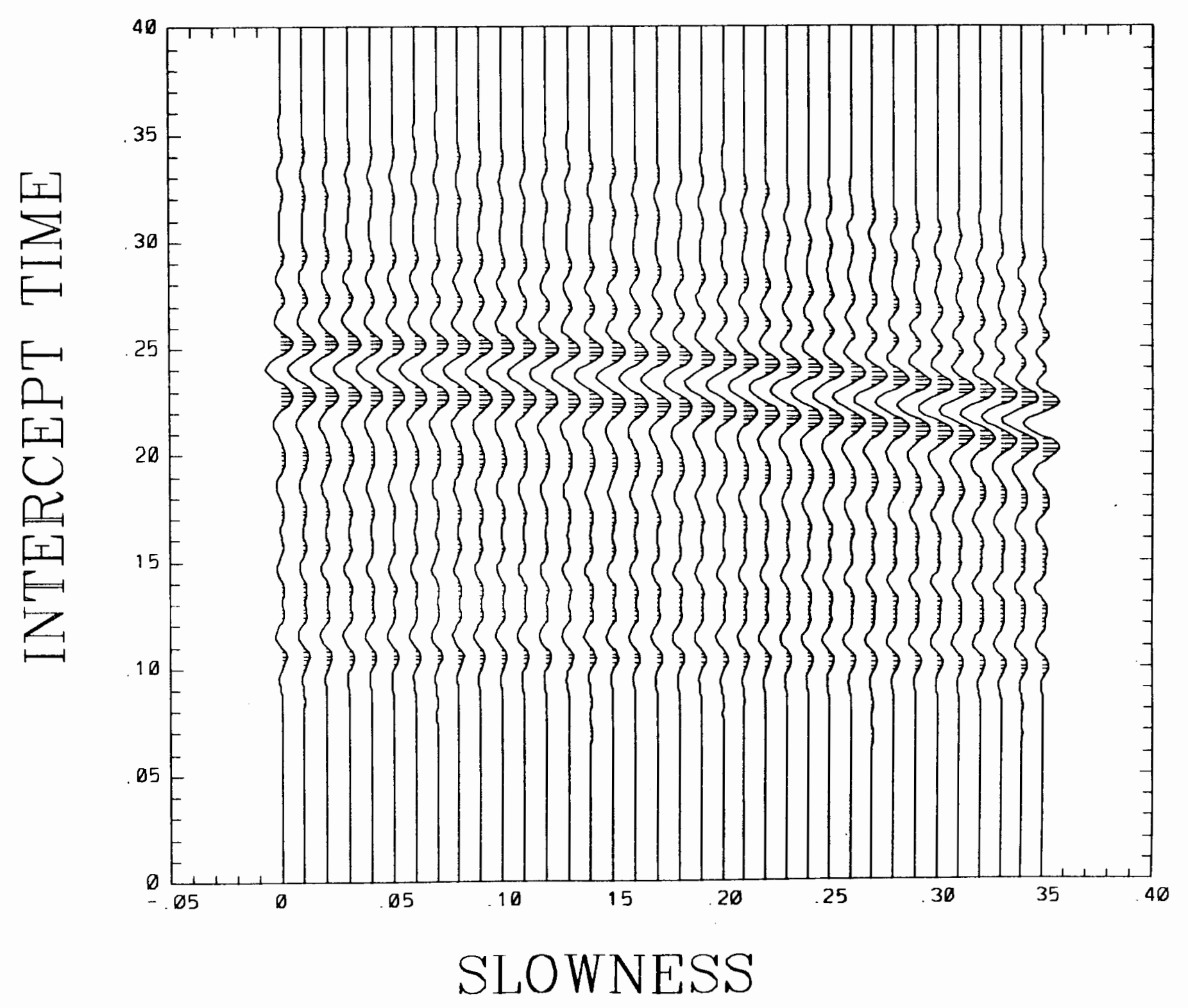




\section{FIGURE 16}

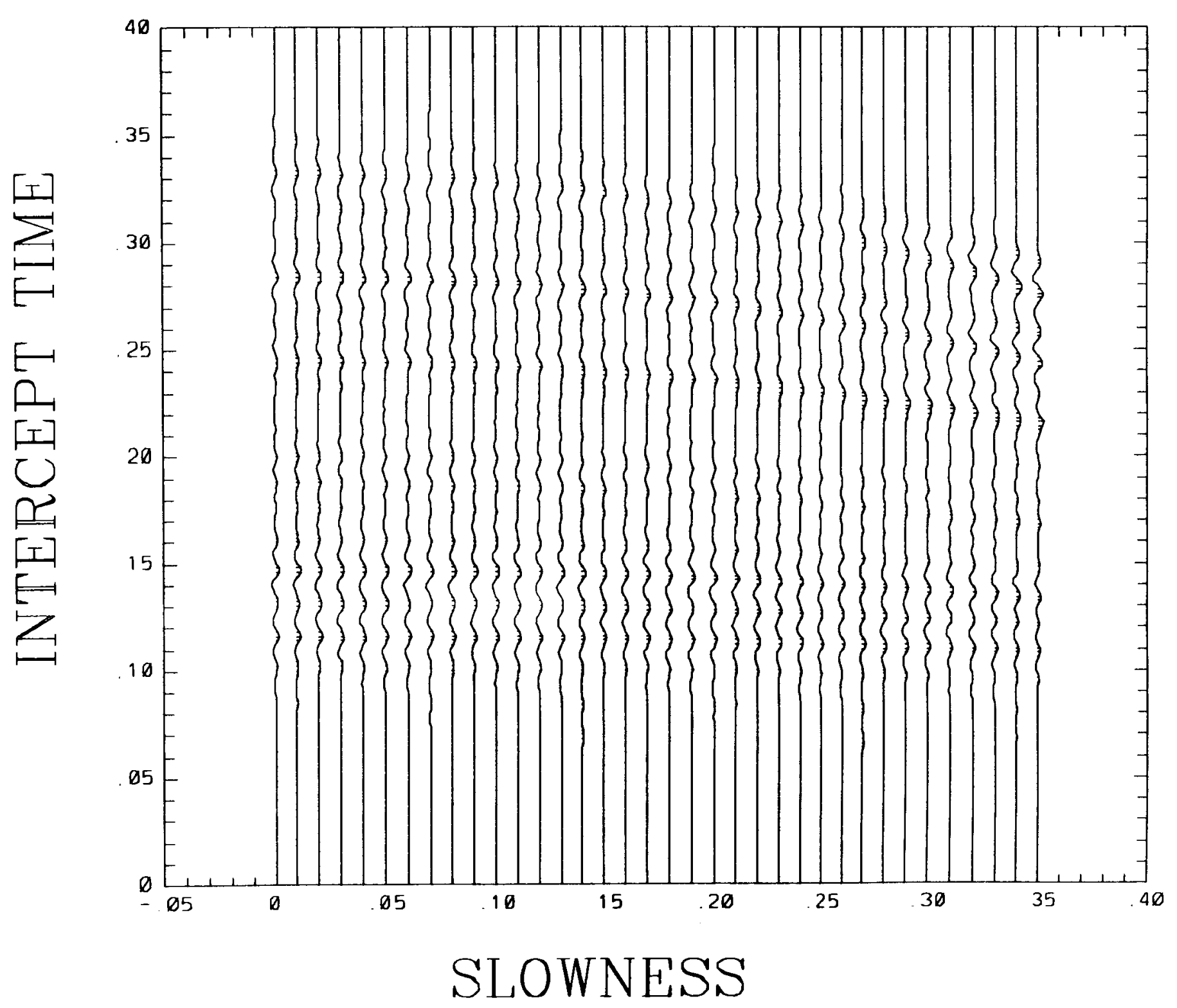

